\title{
CRISTA-NF measurements with unprecedented vertical resolution during the RECONCILE aircraft campaign
}

\author{
J. Ungermann ${ }^{1}$, C. Kalicinsky ${ }^{2}$, F. Olschewski ${ }^{2}$, P. Knieling ${ }^{2}$, L. Hoffmann ${ }^{3}$, J. Blank ${ }^{1}$, W. Woiwode ${ }^{4}$, H. Oelhaf ${ }^{4}$,

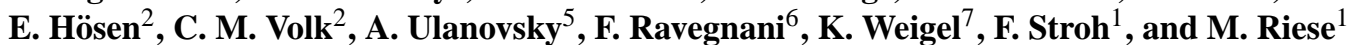 \\ ${ }^{1}$ Institute of Energy and Climate Research - Stratosphere (IEK-7), Research Centre Jülich GmbH, Jülich, Germany \\ ${ }^{2}$ Department of Physics, University of Wuppertal, Wuppertal, Germany \\ ${ }^{3}$ Jülich Supercomputing Centre, Research Centre Jülich GmbH, Jülich, Germany \\ ${ }^{4}$ Institute for Meteorology and Climate Research, Karlsruhe Institute of Technology, Karlsruhe, Germany \\ ${ }^{5}$ Central Aerological Observatory, Dolgoprudny, Russia \\ ${ }^{6}$ Institute of Atmospheric Sciences and Climate, CNR-ISAC, Bologna, Italy \\ ${ }^{7}$ Institute of Environmental Physics (IUP), University of Bremen, Bremen, Germany
}

Correspondence to: J. Ungermann (j.ungermann@fz-juelich.de)

Received: 4 November 2011 - Published in Atmos. Meas. Tech. Discuss.: 21 November 2011

Revised: 23 April 2012 - Accepted: 10 May 2012 - Published: 30 May 2012

\begin{abstract}
The Cryogenic Infrared Spectrometers and Telescope for the Atmosphere - New Frontiers (CRISTA-NF), an airborne infrared limb-sounder, was operated aboard the high-flying Russian research aircraft M55-Geophysica during the Arctic RECONCILE campaign from January to March 2010. This paper describes the calibration process of the instrument and the retrieval algorithm employed and then proceeds to present retrieved trace gas volume mixing ratio cross-sections for one specific flight in this campaign. We are able to resolve the uppermost troposphere/lower stratosphere for several trace gas species for several kilometres below the flight altitude (16 to $19 \mathrm{~km}$ ) with an unprecedented vertical resolution of 400 to $500 \mathrm{~m}$ for the limb-sounding technique. The instrument points sideways with respect to the flight direction. Therefore, the observations are also characterised by a rather high horizontal sampling along the flight track, which provides a full vertical profile every $\approx 15 \mathrm{~km}$. Assembling the vertical trace gas profiles derived from CRISTA-NF measurements to cross-sections shows filaments of vortex and extra-vortex air masses in the vicinity of the polar vortex.

During this campaign, the M55-Geophysica carried further instruments enabling trace gas volume mixing ratios derived from CRISTA-NF to be validated by comparing them with measurements by the in situ instruments HAGAR and FOZAN and observations by MIPAS-STR. This validation suggests that the retrieved trace gas volume mixing ratios are both qualitatively and quantitatively reliable.
\end{abstract}

\section{Introduction}

Passive infrared limb-sounding enables the derivation of trace gas volume mixing ratios and other atmospheric quantities without being in physical contact with the measured air mass. In this way, a vertical profile of atmospheric quantities (e.g., temperature and/or trace gas volume mixing ratios) is reconstructed from a sequence of spectrally resolved radiance measurements taken at different elevation angles from a single observer position. Recent imager instruments (e.g., GLORIA; Friedl-Vallon et al., 2006; Ungermann et al., 2010) measure an increasing amount of such spectra simultaneously instead of sequentially. Due to the observation geometry of limb sounding, emissions by gases in the thermal infrared are summed up over several hundreds of kilometres of air, which makes this technique ideal to detect (trace) gases with small volume mixing ratios or weak emission lines, especially as the cold background of space allows for a high signal to noise ratio. In the past, the technique of infrared limb-sounding was successfully used in several satellite experiments, for example by LIMS (Limb Infrared Monitor of the Stratosphere; Gille and Russel III, 1984), SAMS (Stratospheric and Mesospheric Sounder; Drummond et al., 1980), CRISTA (Cryogenic Infrared Spectrometers and Telescopes for the Atmosphere; Offermann et al., 1999), or MIPAS (Michelson Interferometer for Passive Atmospheric Sounding; Fischer et al., 2008) to provide global trace gas distributions. 
This paper deals with the evaluation of measurements made by an airborne passive infrared limb-sounder, the Cryogenic Infrared Spectrometers and Telescope for the Atmosphere - New Frontiers (CRISTA-NF), which was operated aboard the high-flying Russian research aircraft M55Geophysica. The M55-Geophysica is a well-suited carrier for examining the upper troposphere and lower stratosphere (UTLS) due to its ability to ascend to $\approx 20 \mathrm{~km}$ and its comparatively large load capacity for this altitude. The optical system of CRISTA-NF consists of the centre telescope and two grating spectrometers of the Space Shuttle experiment CRISTA that was successfully flown on the Shuttle Pallet Satellite SPAS in November 1994 [STS 66] and August 1997 [STS 85] (Offermann et al., 1999; Grossmann et al., 2002). CRISTA provided global limb observations of a variety of trace gases with unprecedented horizontal resolution combined with an excellent coverage during its Space Shuttle missions. Numerous small- and medium-scale transport and mixing structures were detected in the observed stratospheric trace gas distributions as a result of pronounced exchange of tropical and extra-tropical air masses in the stratospheric surf zone (e.g. Riese et al., 1999b, 2002).

Aboard M55-Geophysica, CRISTA-NF provides limb observations of trace species in the upper troposphere and lower stratosphere. The UTLS is an atmospheric region characterised by strong gradients and variability in trace gas volume mixing ratios. Many trace gases change their volume mixing ratios by an order of magnitude within a vertical range of a few kilometres when transitioning from tropospheric to stratospheric values (Gettelman et al., 2011). CRISTANF made the first successful observations in this region during the tropical aircraft campaigns SCOUT-O3 (Spang et al., 2008; Hoffmann et al., 2009) and AMMA-SCOUTO3 (Weigel et al., 2010) and observed trace gases like ozone, water vapour, peroxyacetyl nitrate (PAN), $\mathrm{CCl}_{4}$, and $\mathrm{HNO}_{3}$ with the highest two-dimensional resolution to date $(\approx 1 \mathrm{~km}$ vertical $\times 15 \mathrm{~km}$ horizontal along flight track).

This paper describes the evaluation of measurements made by CRISTA-NF during one specific flight in the RECONCILE campaign (Reconciliation of essential process parameters for an enhanced predictability of arctic stratospheric ozone loss and its climate interactions). The RECONCILE campaign took place from January to March 2010. The M55Geophysica, fitted with more than 20 distinct remote-sensing and in situ instruments, performed 12 flights during this time frame. Flight No. 11 (the second flight on 2 March 2010) will be discussed in this paper as an example. This flight was selected because the atmospheric situation was quite diversified and because the sky was cloud-free down to lower altitudes. In addition, data from the MIPAS-STR (Michelson Interferometer for Passive Atmospheric Sounding-STRatospheric aircraft; e.g. Piesch et al., 1996; Höpfner et al., 2000; Keim et al., 2008) instrument is available for this flight, which can be used for improving the retrieval with respect to the pointing of CRISTA-NF (see Sect. 3) and for the validation of retrieval results (see Sect. 7.2.3). The Jülich Rapid Spectral Simulation Code Version 2 (JURASSIC2) is used as forward model and retrieval processor. The Python/C++ based JURASSIC2 and its predecessor were used in several experiments and studies (e.g. Hoffmann et al., 2008; Eckermann et al., 2009; Hoffmann and Alexander, 2009; Ungermann, 2011).

This paper proceeds by describing CRISTA-NF and its calibration in Sect. 2. The setup employed for the retrieval is presented in Sect. 4. The major improvement compared to previous retrievals from CRISTA-NF measurements (e.g. Weigel et al., 2010) is the use of a $250 \mathrm{~m}$ retrieval grid (compared to $500 \mathrm{~m}$ previously) and the corresponding use of all measured spectra of one vertical scan taken at approximately the same spatial sampling. In combination with the small field of view (FOV) of the CRISTA-NF instrument, this allows trace gas volume mixing ratios to be derived with an unprecedented vertical resolution for infrared limb sounders. In Sect. 7, retrieved trace gas volume mixing ratios and corresponding diagnostic information are presented. The results show that the vertical resolution is very good and can also be exploited by reproducing structures of small vertical extent $(\approx 500 \mathrm{~m})$ that are consistent over several consecutive profiles. M55-Geophysica offers unique opportunities for the validation of results, as many instruments employing different measurement techniques were mounted. We exploit this in Sect. 7.2 by comparing our retrieved trace gas volume mixing ratios with in situ measurements of ozone and CFC-11. We also compare our retrieved profiles with profiles retrieved by MIPAS-STR. Compared to CRISTA-NF, MIPAS-STR has a reduced spatial resolution, but a higher spectral resolution, which suggests that their profiles contain less structure, but are probably less affected by interfering species. This permits more reliable retrievals for gases with single spectral lines or narrow features, especially in spectrally dense regions and also allows more minor species to be retrieved (Woiwode et al., 2011).

\section{CRISTA-NF instrument}

CRISTA-NF measures thermal emissions of atmospheric trace gases in the mid-infrared region $(4-15 \mu \mathrm{m})$ using the limb sounding measurement technique. The instrument is operated aboard the Russian high altitude research aircraft M55-Geophysica and has successfully participated in two earlier tropical campaigns. The CRISTA-NF viewing direction is perpendicular to the flight direction of the aircraft looking towards the right-hand side. The Earth's atmosphere is scanned from the flight altitude of the aircraft (up to $20 \mathrm{~km}$ ) down to $5 \mathrm{~km}$ with a vertical sampling of about $250 \mathrm{~m}$ using a Herschel telescope with a tiltable mirror. At each corresponding tangent point the spectral range from $4-15 \mu \mathrm{m}$ is scanned by two Ebert-Fastie grating spectrometers (e.g. Fastie, 1991) that allow spectrally resolved measurements 
of the incoming radiance with different spectral resolution $(\lambda / \Delta \lambda \sim 1000$ and 500, respectively). For the detection of the radiance, a number of cryogenic semiconductor detectors are used and operated at very low temperatures of about $13 \mathrm{~K}$. A detailed description of the optical system and the cryostat of CRISTA-NF is given by Kullmann et al. (2004).

Due to the low temperatures of the detectors and the whole optical system of the instrument, a good signal-to-noise ratio and a high measurement speed (1.2 s per spectrum) can be obtained. According to the speed of the aircraft and the fast measurement time of $70 \mathrm{~s}$ per vertical scan (60 altitude steps/spectra), the horizontal sampling along the flight direction is about $15 \mathrm{~km}$. The aperture of the optical systems provides a FOV of 3 arcmin $\times 30$ arcmin (vertical times horizontal) resulting in a vertical extent of $\approx 300 \mathrm{~m}$ at $10 \mathrm{~km}$ tangent height (Spang et al., 2008). The combination of this comparatively small vertical FOV and the fine vertical sampling provides the basis for the high vertical resolution discussed in Sect. 7.1. In combination with the dense horizontal sampling along the flight track (resulting from the fast measurement speed), two-dimensional trace gas distributions with high spatial resolution are obtained in the UTLS (hereafter termed "cross-sections").

\section{Calibration and level 0/1 processing}

The calibration of CRISTA-NF comprises three major procedures: a wavelength calibration, a radiometric absolute calibration and a line-of-sight (LOS) calibration (see Schroeder et al., 2009). The wavelength calibration is used to convert the output voltages of the gratings used in the Ebert-Fastie spectrometers to wavelength values. The calibration is based on laboratory measurements of absorption spectra of infrared active gases. Since the absorption measurements in the laboratory are not accurate enough for highly precise retrievals, they are complemented by a post-flight calibration based on atmospheric emission spectra obtained during the observations onboard M55-Geophysica. The detector raw signals (output voltages) are converted to limb radiance spectra by means of calibration parameters obtained from the radiometric absolute calibration utilising black-body emission spectra. The tangent height of each spectrum measured during the aircraft flights is calculated based on parameters obtained from the LOS calibration.

The post-flight wavelength calibration is based on comparisons between these measured spectra and simulated spectra using the Reference Forward Model (RFM; Dudhia et al., 2002). By this approach, updated calibration parameters are determined for the calculation of the wavelength from the recorded grating voltages. As initial input data for the RFM, we use a combination of CLaMS (Chemical Lagrangian Model of the Stratosphere; e.g. Grooß et al., 2005) model data and the climatology of Remedios et al. (2007) in order to obtain a realistic atmospheric state. After a first retrieval, the obtained atmospheric profiles replace the model data for a further (final) refinement of the calibration parameters.

In order to calculate the LOS, accurate knowledge of the M55-Geophysica roll and pitch angles is required. Since the angle information of the M55-Geophysica determined by its inertial navigation system and provided by the UCSE (unit for connection with scientific equipment) is not accurate enough for our purposes, we decided to use the very accurate measurements of these quantities by the MIPAS-STR attitude system instead (Keim, 2002). The MIPAS-STR attitude system AHRS (Attitude and Heading Reference System) is rotated by about $90^{\circ}$ with respect to the M55-Geophysica attitude system. Therefore, the MIPAS-STR pitch angle corresponds to the M55-Geophysica roll angle and vice versa (to a very good approximation). Angle offsets between the MIPAS-STR and the CRISTA-NF instrument caused by the integration of the two instruments are taken into account and the angle information is corrected to the reference system of the CRISTA-NF instrument.

The other quantities (yaw angle, observer location), needed to determine the LOS, are taken from the M55Geophysica UCSE recordings. Hence, an obvious time shift between the MIPAS-STR and the UCSE recordings is corrected to obtain a compatible set of quantities.

Comparisons between two retrieval results, the first obtained using M55-Geophysica attitude data only and the other using the combination of MIPAS-STR roll and pitch angle information and other quantities from the M55Geophysica attitude system, show better agreement of simulated and measured spectra and less noisy behaviour of retrieved quantities for the second set of attitude information. This confirms our assumption that the utilisation of MIPAS-STR data for the M55-Geophysica roll and pitch angle improves the retrieval results.

The atmospheric radiance enters the CRISTA-NF optical system through a zinc selenide ( $\mathrm{ZnSe}$ ) window, which separates the instrument from the surrounding atmosphere. The emissivity and transmissivity of this window were determined during the calibration procedure of the AMMASCOUT-O3 campaign (see Schroeder et al., 2009) and were used to account for the influence of the $\mathrm{ZnSe}$ window on our radiometric calibration. Calibrated limb radiance values are shown in Fig. 1 for a specific detector channel (Low Resolution Spectrometer channel 6). This channel covers the spectral range from 776.0 to $868.0 \mathrm{~cm}^{-1}$ and is used for the retrievals discussed in the following sections.

The CRISTA-NF detectors show a non-stationary behaviour after illumination changes, which is called detector relaxation. It depends on the illumination intensity as well as on the illumination history. Detector relaxations are, therefore, also a function of the spectral scanning direction of the gratings, which is reversed after each altitude (tangent height) step (e.g. Riese et al., 1999a). In order to asses the impact of this effect on the limb radiance spectra measured during RECONCILE, altitude profiles of the integrated radiance 


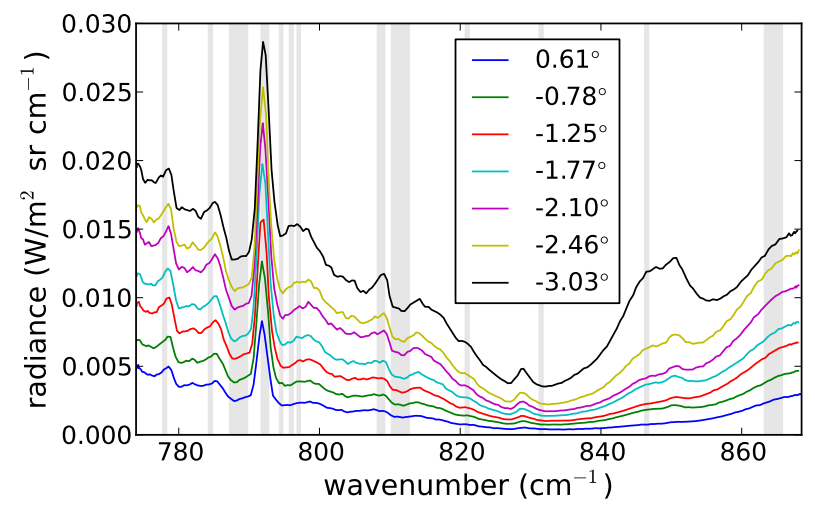

Fig. 1. Seven representative calibrated spectra as measured by the LRS6 detector at different elevation angles (negative angles point downward). The frequency ranges of the IMWs used for the retrieval are shown as grey boxes. With an observer altitude of $19.1 \mathrm{~km}$, the angle of $-0.78^{\circ}$ corresponds to a tangent point altitude of $18.41 \mathrm{~km}$ while the angle of $-3.03^{\circ}$ corresponds to a tangent altitude of $10.1 \mathrm{~km}$.

in three representative integrated microwindows (IMW) are shown in Fig. 2 separately for forward and backward spectra. Fortunately, only negligible systematic differences between the two spectra types are visible in these IMWs for the observation conditions encountered during RECONCILE and the employed wavelength calibration. The other IMWs used in the retrieval (not shown) exhibit the same behaviour. Hence, we can use both spectra types simultaneously, which provides twice as good vertical sampling compared to that used by Weigel et al. (2010) under conditions where small relaxation effects were evident. Therefore, The RECONCILE observations allow, for the first time, the full potential of the combination of a $250 \mathrm{~m}$ vertical sampling and a comparably small vertical FOV to be explored in terms of achievable vertical resolution (see Sect. 7.1).

For the retrieval, the measured limb radiance spectra (level-1 data) are filtered with respect to clouds or optically thick conditions. The so called cloud index (CI) is a good quantity for this issue. It is defined as the ratio between the integrated radiances in two specific IMWs. The first from 791.0 to $793.0 \mathrm{~cm}^{-1}$ is dominated by $\mathrm{CO}_{2}$ emissions, whereas the second from 832.0 to $834.0 \mathrm{~cm}^{-1}$ is located in an atmospheric window region (see Spang et al., 2008). A low CI value indicates the presence of clouds or optically thick conditions due to aerosol background or the water vapour continuum. The threshold for the filtering is set to a value of 3.5 to exclude all spectra influenced by clouds, etc. from the retrieval.

\section{Retrieval}

Here, retrieval is the process of deriving atmospheric constituents from limb-radiance measurements. This is an ill-

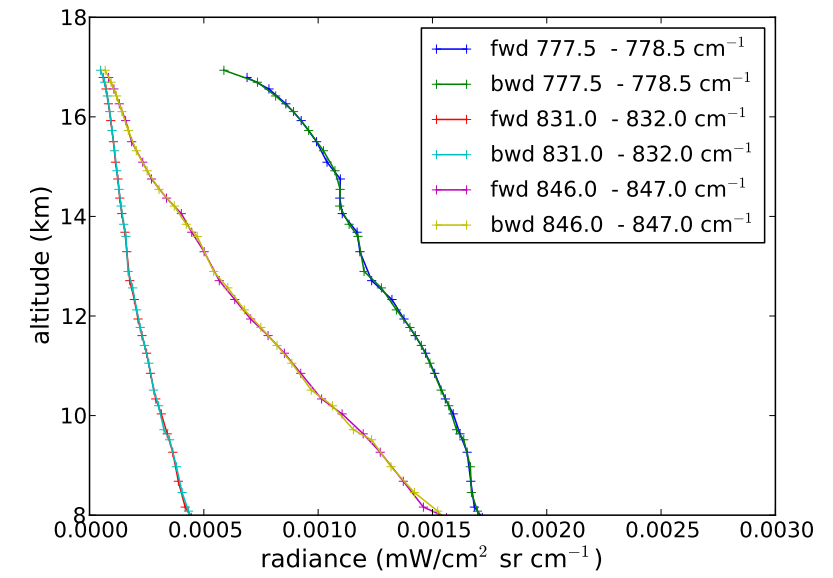

Fig. 2. Altitude profiles of the integrated radiances in three different integrated microwindows $\left(777.5\right.$ to $778.5 \mathrm{~cm}^{-1}, 831.0$ to $832.0 \mathrm{~cm}^{-1}, 846.0$ to $847.0 \mathrm{~cm}^{-1}$ ) separated into forward and backward spectra.

posed inverse problem, which can be solved by means of a forward model simulating the radiative transport and the instrument, on the one hand, and a nonlinear minimisation on the other. Let $\mathbf{F}: \mathbb{R}^{n} \mapsto \mathbb{R}^{m}$ be a forward model that maps an $n$-dimensional discrete representation of the atmosphere $\boldsymbol{x} \in \mathbb{R}^{n}$ (here: a vertical profile) including temperature, pressure, aerosol (or extinction) and trace gas volume mixing ratios onto a set of $m$ radiance measurements $\boldsymbol{y} \in \mathbb{R}^{m}$. The inverse problem now consists of finding an $\boldsymbol{x}$ that is (approximately) mapped by $\mathbf{F}$ onto measured radiances $\boldsymbol{y}$. This is problematic as a straightforward inversion is often very sensitive to small changes of the measured radiances caused by noise (or similar effects).

One way to find a stable solution is minimising a cost function $J: \mathbb{R}^{n} \mapsto \mathbb{R}$ :

$$
\begin{aligned}
J(\boldsymbol{x}) & =(\mathbf{F}(\boldsymbol{x})-\boldsymbol{y}){ }^{\mathrm{T}} \mathbf{S}_{\epsilon}^{-1}(\mathbf{F}(\boldsymbol{x})-\boldsymbol{y}) \\
& +\left(\boldsymbol{x}-\boldsymbol{x}_{\mathrm{a}}\right)^{\mathrm{T}} \mathbf{S}_{\mathrm{a}}^{-1}\left(\boldsymbol{x}-\boldsymbol{x}_{\mathrm{a}}\right) .
\end{aligned}
$$

The matrix $\mathbf{S}_{\epsilon} \in \mathbb{R}^{m \times m}$ is the covariance matrix of the measurement errors. We assume an uncorrelated error budget of $1 \%$ for the retrieval as a simple approximation to the true covariance matrix. The second term in $J$ (right side of Eq. 1) is a regularisation term, which implies that minimising this cost function is a well-posed approximation to the original ill-posed inverse problem. The regularisation constraint stabilises the solution with respect to measurement errors and allows the introduction of a priori knowledge about the atmosphere. The setup of the a priori vector $\boldsymbol{x}_{\mathrm{a}} \in \mathbb{R}^{n}$ is described in Sect. 4.2, while the setup of the regularisation matrix is described in Sect. 4.3. The cost function is minimised by a truncated quasi-Newton iteration formula employing an adjoint model to calculate the required Jacobian matrix $\mathbf{F}^{\prime}$ of the forward model (Ungermann et al., 2010). 


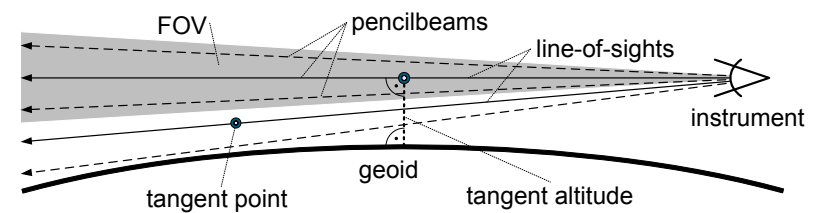

Fig. 3. Diagram of observation geometry. The CRISTA-NF instrument is located to the right. Line-of-sight of radiance measurements are shown as straight lines. Additional pencilbeams are shown as dotted lines. The FOV of one measurement is shown as a grey cone (not to scale).

\subsection{Radiative transfer model}

A single radiative transfer calculation of our forward model simulates an infinitesimally thin beam of radiation along the LOS, also called a "pencilbeam", which neglects the effects of FOV, etc. Each pencilbeam maps the atmospheric state onto a single radiance value.

Each pencilbeam is determined by an observer position, a viewing direction and a spectral range. First, a ray-tracing step is performed using a 3-D ray-tracing routine (Hase and Höpfner, 1999) that determines the LOS. Using this interpolated LOS, the emissivity growth approximation method (EGA; e.g. Weinreb and Neuendorffer, 1973; Gordley and Russell, 1981) and the Curtis-Godson approximation (CGA; Curtis, 1952; Godson, 1953) are each used to approximate the emission and absorption processes along the LOS to determine incoming radiances. Both methods allow for easy computing of the total transmissivity between the instrument and any point on the discretised LOS, which avoids the summation of errors if only the emissivities of short segments were calculated and multiplied. The radiances derived from the two methods are in turn combined using a simple regression scheme to minimise the deviation to a more exact lineby-line model (Weigel et al., 2010). The combined method is typically subjected to smaller systematic errors than either the CGA or the EGA method alone (e.g. Francis et al., 2006). Compared to conventional line-by-line calculations, the EGA and CGA methods are faster by a factor of about 1000 , since the radiative transfer is based on pre-calculated, spectrally averaged values of emissivity stored in look-up tables. The emissivity look-up tables for the forward model are prepared by means of exact line-by-line calculations utilising the RFM. We use the spectroscopic data from HITRAN 2008 (HIgh resolution TRANsmission; Rothman et al., 2009) including all updates up to August 2011 to generate the tables used for the following retrievals.

Each measurement is affected by the FOV of the instrument, which can be approximated by a Gaussian with an FWHM (full width at half maximum) of about 3 arcmin for CRISTA-NF (identical to CRISTA; Riese et al., 1999a). To properly accommodate this effect, the convolution of the weighting function with incoming radiation is numerically

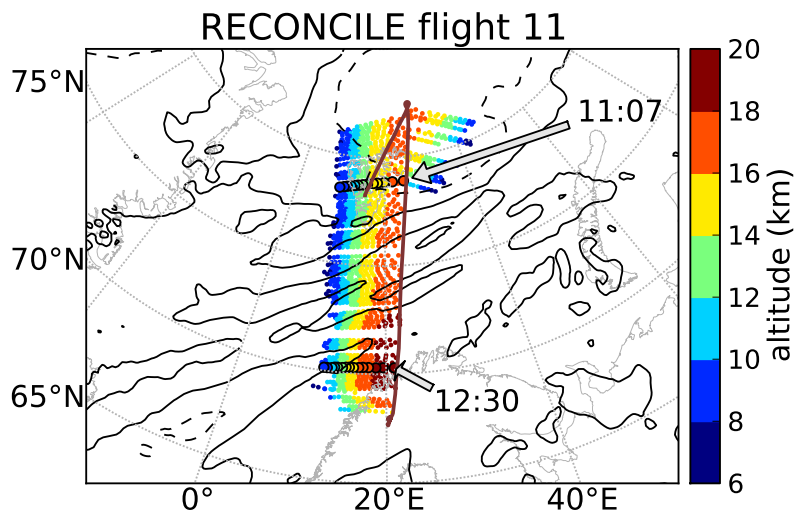

Fig. 4. Plot of tangent point locations of CRISTA-NF measurements taken during RECONCILE flight 11, the second flight of 2 March 2011. The flight path is drawn in dark red. The Locations of every fourth tangent point are drawn as circles with colour code according to tangent point altitude. Thick black contour lines indicate a potential vorticity of $20 \mathrm{PVU}$ at $17 \mathrm{~km}$ at 12:00 UTC (indicating the position and orientation of filaments across the flight path and dashed black contour lines show 26 PVU at the same altitude and time (roughly indicating the location of the polar vortex core).

integrated, which implies the casting of several pencilbeams per observation at different elevation angles. This is illustrated in Fig. 3. In this example, the radiance of the upper measurement would be computed as the weighted average of the radiances computed by the upper three pencilbeams.

The primary retrieval targets of our analysis are $\mathrm{CCl}_{4}$, CFC-11, $\mathrm{H}_{2} \mathrm{O}, \mathrm{HNO}_{3}, \mathrm{O}_{3}$ and $\mathrm{ClONO}_{2}$. Secondary retrieval targets are aerosol, temperature, PAN, CFC-113 and HCFC22. These latter trace gases are mainly included in the retrieval process to minimise their impact on uncertainties in the primary retrieval targets. In addition, contributions of $\mathrm{CO}_{2}, \mathrm{HNO}_{4}$, OCS and CFC-114 are taken into account when calculating limb-radiance values. However, the volume mixing ratios of these non-retrieved trace gases are fixed to climatological values. Temperature, aerosol, $\mathrm{CCl}_{4}, \mathrm{CFC}-11$, $\mathrm{H}_{2} \mathrm{O}$, PAN, CFC-113 and HCFC-22 are retrieved in the altitude range from 0 to $25 \mathrm{~km}$ while $\mathrm{HNO}_{3}, \mathrm{O}_{3}$ and $\mathrm{ClONO}_{2}$ are retrieved between 0 and $65 \mathrm{~km}$.

Table 1 summarises the IMWs employed in the following retrievals. They are also depicted as grey boxes in Fig. 1. The selection of these IMWs is largely identical with the setup previously used by Weigel et al. (2010). In addition, we included a frequency range for the retrieval of $\mathrm{ClONO}_{2}$, which was previously successfully used for CRISTA satellite retrievals (Riese et al., 1997, 1999a), and three frequencies to derive the secondary retrieval targets CFC-113 and HCFC22. We do not spectrally resolve radiance within an IMW, but use only one integrated radiance value.

As described above, the forward model is improved by a simple regression scheme. In a first pass, the flight is evaluated without the regression by simply averaging the limb 
Table 1. Microwindows used in the retrieval including main targets. The last two columns give the standard deviation of relative error of our forward model compared to RFM with and without regression.

\begin{tabular}{cclcc}
\hline $\begin{array}{c}\text { micro- } \\
\text { window }\end{array}$ & $\begin{array}{c}\text { spectral } \\
\text { range }\left(\mathrm{cm}^{-1}\right)\end{array}$ & $\begin{array}{l}\text { main } \\
\text { target }\end{array}$ & $\begin{array}{c}\text { error } \\
\text { w/o regr. }(\%)\end{array}$ & $\begin{array}{c}\text { error } \\
\text { w/regr. (\%) }\end{array}$ \\
\hline 0 & $777.5-778.5$ & $\mathrm{O}_{3}$ & 0.56 & 0.05 \\
1 & $784.0-785.0$ & $\mathrm{H}_{2} \mathrm{O}$ & 0.45 & 0.07 \\
2 & $787.0-790.0$ & offset & 0.36 & 0.06 \\
3 & $791.5-793.0$ & temperature & 0.22 & 0.03 \\
4 & $794.1-795.0$ & $\mathrm{PAN}_{3}$ & 0.37 & 0.08 \\
5 & $795.5-796.5$ & $\mathrm{O}_{3}$ & 0.41 & 0.08 \\
6 & $796.6-797.5$ & $\mathrm{CCl}_{4}$ & 0.70 & 0.08 \\
7 & $808.0-809.0$ & $\mathrm{HCFC}-22_{2}$ & 0.36 & 0.08 \\
8 & $810.0-813.0$ & $\mathrm{ClONO}_{2}$ & 0.33 & 0.08 \\
9 & $820.5-821.5$ & $\mathrm{HCFC}_{2} 22$ & 0.27 & 0.12 \\
10 & $831.0-832.0$ & aerosol & 0.24 & 0.22 \\
11 & $846.0-847.0$ & $\mathrm{CFC}^{2} 11$ & 0.11 & 0.10 \\
12 & $863.0-866.0$ & $\mathrm{HNO}_{3}$ & 0.10 & 0.04 \\
\hline
\end{tabular}

radiances as calculated by the CGA and EGA method. From the retrieved profiles, a subset of 30 well-converged profiles is selected at roughly regular temporal intervals. Using these profiles, the regression scheme is trained using the method of Weigel et al. (2010). The flight is then re-evaluated using the regression parameters derived in this way. Table 1 shows the standard deviation per IMW between our forward model and the RFM with and without regression correction for all retrieved profiles. The regression reduces the standard deviation of model difference from up to $1 \%$ down to at most $0.22 \%$. In addition, the mean bias (not listed) is reduced from up to $1.2 \%$ down to comparatively negligible $0.02 \%$.

In contrast to previous CRISTA-NF studies, we employ the full vertical sampling $(\approx 250 \mathrm{~m})$ in our retrieval by combining measurements for both scanning directions of the spectrometer grating (see also Sect. 3).

The retrieval grid has a fixed spacing of $250 \mathrm{~m}$ below $20 \mathrm{~km}$ and, thereby, roughly follows the typical tangent point altitude distance. Even though upward-looking spectra are taken and used, it is not expected to attain this fine resolution between aircraft altitude and $20 \mathrm{~km}$. Above $20 \mathrm{~km}$, the retrieval grid distance is increased to $1 \mathrm{~km}$, followed by a second increase to $2 \mathrm{~km}$ above $30 \mathrm{~km}$. The highest retrieval grid point is located at $66 \mathrm{~km}$. All altitudes mentioned are geometric altitudes.

\subsection{A priori and model data}

The regularisation requires an atmospheric state vector $\boldsymbol{x}_{\mathrm{a}}$ and also standard deviations for all involved quantities. We use three sources for these parameters.

The first important source of a priori data is the ECMWF ERA-Interim (Dee et al., 2011) data, which supplies temperature, pressure and water vapour on a 3-D grid with horizontal resolution of $1.125^{\circ}$ and a temporal sampling of $6 \mathrm{~h}$. As ECMWF does not supply standard deviations for the model data, these need to be taken from a different source. For the primary target water vapour, we conservatively use the standard deviations supplied by Remedios et al. (2007). For the secondary target temperature, a comparably small standard deviation of $1 \mathrm{~K}$ is used. We do not retrieve pressure as we believe the pressure data supplied by ECMWF to be of high quality. For error analysis, we assume a standard deviation of $0.1 \%$ of given pressure values, which indeed leads to a negligible error contribution.

For the secondary retrieval target PAN, we use a zeroprofile as an a priori value and a priori standard deviations as estimated by Glatthor et al. (2007).

For the other trace gases, we use the climatology of Remedios et al. (2007), which is currently the most comprehensive climatology with respect to number of contained trace gas species. It contains both mean values and associated standard deviations for all our retrieval targets and background gases. The mean values for $\mathrm{CO}_{2}$ were adjusted for the typical annual increase. Since this climatology has several shortcomings, e.g., too small mean values for HCFC22 and CFC-113 (Dufour et al., 2005; Weigel et al., 2010), we decided to make these gases secondary retrieval targets, whereas previous CRISTA-NF studies did not retrieve them.

\subsection{Regularisation matrix}

We use a Tikhonov regularisation (Tikhonov and Arsenin, 1977) to assemble the a priori covariance matrix $\mathbf{S}_{\mathrm{a}}^{-1}$. The simplest choice for the Tikhonov regularisation matrix is an identity matrix. In combination with an a priori vector $\boldsymbol{x}_{\mathrm{a}}$, this selects the solution $\boldsymbol{x}$ with the smallest norm $\left\|\boldsymbol{x}-\boldsymbol{x}_{\mathrm{a}}\right\|_{2}$ from all possible solutions. Also higher-order derivatives may be constrained (Twomey, 1977). Here, a weighted combination of the two approaches is used that is more flexible than either alone.

Two matrices $\mathbf{L}_{0}$ and $\mathbf{L}_{1} \in \mathbb{R}^{n \times n}$ corresponding to regularisation of the absolute value and the first order derivative are defined. Then $\mathbf{S}_{\mathrm{a}}^{-1}$ is set to

$\mathbf{S}_{\mathrm{a}}^{-1}=\left(\alpha_{0}\right)^{2} \mathbf{L}_{0}^{\mathrm{T}} \mathbf{L}_{0}+\left(\alpha_{1}\right)^{2} \mathbf{L}_{1}^{\mathrm{T}} \mathbf{L}_{1}$

with the tuning parameters $\alpha_{0}$ and $\alpha_{1} \in \mathbb{R}$. Let $\mathbf{M} \in \mathbb{R}^{n \times n}$ be a diagonal matrix with the reciprocal values of the retrieval quantity standard deviations $\sigma_{i}$ on its diagonal. We then define $\mathbf{L}_{0}=\mathbf{M}$.

The $\mathbf{L}_{1}$ matrix is a simple Tikhonov regularisation matrix of the first order, adjusted for the non-uniform grid size and weighted with the standard deviations of the retrieval targets. For a 1-D single target retrieval, let $h_{i}$ denote the grid distance between vector element $i$ and $i+1$ of the atmospheric state $\boldsymbol{x}$. Then, let the scaling matrix $\mathbf{N} \in \mathbb{R}^{n \times n}$ be defined as a diagonal matrix with $\sqrt{c_{\mathrm{q}} / h_{i}}$ on its diagonal, where $c_{\mathrm{q}} \in \mathbb{R}$ defines a quantity-specific correlation length. With these diagonal helper matrices, the $\mathbf{L}_{1}$ matrix would be defined as 
$\mathbf{L}_{1}=\frac{\mathbf{M} \cdot \mathbf{N}}{\sqrt{2}}\left(\begin{array}{ccccc}-1 & 1 & 0 & \ldots & 0 \\ 0 & -1 & 1 & \ddots & \vdots \\ \vdots & \ddots & \ddots & \ddots & 0 \\ 0 & \ldots & 0 & -1 & 1 \\ 0 & \ldots & 0 & 0 & 0\end{array}\right)$

For $c_{\mathrm{q}}=1$ and without the scaling by $\mathbf{M},\left\|\mathbf{L}_{1} \boldsymbol{x}\right\|$ approximates the Euclidean norm of the first order derivative of the continuous function sampled by the state vector $\boldsymbol{x}$. The scaling by $\mathbf{M}$ gives a simple altitude-dependent regularisation that weakens the regularisation if a quantity is more variable at that specific altitude than at other heights. For $\alpha_{0}=\alpha_{1}=1$, this Tikhonov regularisation is very similar to the auto-regressive approach in optimal estimation (e.g. Steck and von Clarmann, 2001). Indeed, we found that using $c_{\mathrm{q}}=\alpha_{0}=\alpha_{1}=1$ already delivers very useful results. For multi-target retrievals, $\mathbf{L}_{1}$ is assembled as a block diagonal matrix with one block for each target, each defined as described above. The correlation lengths chosen for the presented results are collected in Table 2.

As a bias of the absolute values of the solution $\boldsymbol{x}_{\mathrm{f}}$ towards a priori vector $\boldsymbol{x}_{\mathrm{a}}$ is quite often seen as a problem, we reduced $\alpha_{0}$ to $\sqrt{0.1}$. This corresponds to a tenfold increase of the variances for the regularisation with respect to absolute value. Reducing $\alpha_{0}$ further was detrimental to the convergence of some profiles. However, with the strength of $\sqrt{0.1}$, the bias in the results apparent by evaluating the measurement contribution (see also Sect. 5) is removed, while retaining a stable convergence and rather well-conditioned equation system matrices.

\section{Diagnostics}

An important diagnostic tool is the averaging kernel matrix $\mathbf{A} \in \mathbb{R}^{n \times n}$ that maps the true atmospheric state $\boldsymbol{x}_{\mathrm{t}} \in \mathbb{R}^{n}$ onto the retrieval result (neglecting the influence of a priori information and noise). It consists of the matrix product between the gain matrix $\mathbf{G} \in \mathbb{R}^{n \times m}$ and the Jacobian matrix $\mathbf{F}^{\prime}\left(\boldsymbol{x}_{\mathrm{f}}\right)$ evaluated at the solution $\boldsymbol{x}_{\mathrm{f}}$ with $\mathbf{G}$ defined as

$\mathbf{G}=\left(\mathbf{S}_{\mathrm{a}}^{-1}+\mathbf{F}^{\prime}\left(\boldsymbol{x}_{\mathrm{f}}\right)^{\mathrm{T}} \mathbf{S}_{\epsilon}^{-1} \mathbf{F}^{\prime}\left(\boldsymbol{x}_{\mathrm{f}}\right)\right)^{-1} \mathbf{F}^{\prime}\left(\boldsymbol{x}_{\mathrm{f}}\right)^{\mathrm{T}} \mathbf{S}_{\epsilon}^{-1}$

and consequently

$$
\begin{aligned}
\mathbf{A} & =\left(\mathbf{S}_{\mathrm{a}}^{-1}+\mathbf{F}^{\prime}\left(\boldsymbol{x}_{\mathrm{f}}\right)^{\mathrm{T}} \mathbf{S}_{\epsilon}^{-1} \mathbf{F}^{\prime}\left(\boldsymbol{x}_{\mathrm{f}}\right)\right)^{-1} \mathbf{F}^{\prime}\left(\boldsymbol{x}_{\mathrm{f}}\right)^{\mathrm{T}} \mathbf{S}_{\epsilon}^{-1} \mathbf{F}^{\prime}\left(\boldsymbol{x}_{\mathrm{f}}\right) \\
& =\mathbf{G F}^{\prime}\left(\boldsymbol{x}_{\mathrm{f}}\right) .
\end{aligned}
$$

The averaging kernel matrix can be analysed to derive useful quantities like measurement contribution or resolution. The measurement contribution is calculated by summing up all elements of a row of the averaging kernel matrix, which typically gives a value between zero and one. This value indicates the influence of a priori information of zeroth order on the retrieval result (a value of "1" indicates a high measurement contribution and a value of " 0 " indicates a high a priori contribution). This paper employs the FWHM of the averaging kernel matrix rows as measure of vertical resolution (e.g. Rodgers, 2000, p.61f).

The usual averaging kernel matrix does not contain enough information to deduce the horizontal resolution along the LOS. Instead of the map between the 1-D true state and the 1-D retrieval result, a map between the 2-D true state and the 1-D result is required to capture the spatial dimension along the LOS. This can be straightforwardly generated by extending the 1-D retrieval solution $\boldsymbol{x}_{\mathrm{f}}$ horizontally into a 2-D plane $\tilde{\boldsymbol{x}}_{\mathrm{f}}$ in the along-sight direction and calculating the Jacobian matrix $\tilde{\mathbf{F}}^{\prime}\left(\tilde{\boldsymbol{x}}_{\mathrm{f}}\right)$ of a 2-D forward model $\tilde{\boldsymbol{F}}$ with respect to this 2-D representation. The diagnostic formula

$\boldsymbol{x}_{\mathrm{f}}=\mathbf{G} \tilde{\mathbf{F}}^{\prime}\left(\tilde{\boldsymbol{x}}_{\mathrm{f}}\right) \tilde{\boldsymbol{x}}_{\mathrm{t}}+(\mathbf{I}-\mathbf{A}) \boldsymbol{x}_{\mathrm{a}}+\mathbf{G} \epsilon$,

then allows the horizontal resolution to be deduced along the LOS or a better comparison against model data by evaluating the matrix $\mathbf{G} \tilde{\mathbf{F}}^{\prime}\left(\tilde{\boldsymbol{x}}_{\mathrm{f}}\right)$ (Ungermann et al., 2011). This approach is equivalent to performing a 2-D retrieval that enforces horizontal homogeneity as employed by von Clarmann et al. (2009). The resulting resolution calculated in this way is often larger than the typically used figure of FWHM within the tangent altitude layer only, as it also includes horizontal smoothing stemming from higher layers. This is similar to the typical vertical resolution, which always includes the horizontal component.

To derive error estimates for the result, the gain matrix $\mathbf{G}$ is the most important tool, as it can be used to convert an uncertainty in the measurement domain into an uncertainty in the retrieval result. In this way, the effect of instrument errors can be easily calculated as

$\mathbf{S}_{\text {instrument }}=\mathbf{G S}_{\epsilon} \mathbf{G}^{\mathrm{T}}$.

In a similar manner, the effect of the uncertainty in background gases can be estimated by first assembling a covariance matrix describing (an estimate of) the known distribution of the gas in question. This matrix is then converted into a covariance matrix describing the following uncertainty in the measurements by multiplications by the Jacobian matrix of the forward model with respect to this trace gas. For example, let $\mathbf{S}_{\mathrm{HNO}_{4}}$ be the covariance matrix of the $\mathrm{HNO}_{4}$ distribution, which is one of our unretrieved background gases; to assemble this matrix, it is necessary to make some assumption about vertical correlation length, which is not contained in the employed climatology. Let $\boldsymbol{x}_{\mathrm{HNO}_{4}}$ be our best estimate of the $\mathrm{HNO}_{4}$ volume mixing ratios present during the time of our measurement. Let $\mathbf{F}^{\prime}\left(\boldsymbol{x}_{\mathrm{HNO}_{4}}\right)$ be the Jacobian matrix of the forward model with respect to $\mathrm{HNO}_{4}$. The effect of the uncertainty of $\mathrm{HNO}_{4}$ volume mixing ratio on the retrieval result can then be estimated as

$\mathbf{S}_{\text {error by } \mathrm{HNO}_{4}}=\mathbf{G F}^{\prime}\left(\boldsymbol{x}_{\mathrm{HNO}_{4}}\right) \mathbf{S}_{\mathrm{HNO}_{4}} \mathbf{F}^{\prime}\left(\boldsymbol{x}_{\mathrm{HNO}_{4}}\right)^{\mathrm{T}} \mathbf{G}^{\mathrm{T}}$. 
Table 2. Parameters employed for the regularisation: Tikhonov strength and correlation lengths.

\begin{tabular}{lr}
\hline parameter & value \\
\hline$\alpha_{0}$ & $\sqrt{0.1}$ \\
$\alpha_{1}$ & 1 \\
$c_{\text {temperature }}$ & $2 \mathrm{~km}$ \\
$c_{\text {aerosol }}$ & $64 \mathrm{~km}$ \\
$c_{\mathrm{CCl} 4}$ & $1 \mathrm{~km}$ \\
$c_{\mathrm{CFC}-11}$ & $0.25 \mathrm{~km}$ \\
$c_{\mathrm{CFC}-113}$ & $8 \mathrm{~km}$ \\
$c_{\mathrm{ClONO}}$ & $2 \mathrm{~km}$ \\
$c_{\mathrm{H}_{2} \mathrm{O}}$ & $2 \mathrm{~km}$ \\
$c_{\mathrm{HCFC}-22}$ & $8 \mathrm{~km}$ \\
$c_{\mathrm{HNO}}$ & $2 \mathrm{~km}$ \\
$c_{\mathrm{O}_{3}}$ & $4 \mathrm{~km}$ \\
$c_{\mathrm{PAN}}$ & $8 \mathrm{~km}$ \\
\hline
\end{tabular}

The effect of random elevation angle errors of individual spectra is covered by the error budget of $1 \%$ per measurement. In addition, a potential systematic elevation angle offset error consistent over all spectra of one vertical scan is treated separately. The absolute error in retrieved trace gas volume mixing ratios induced by such a constant elevation angle offset is especially large in the presence of strong vertical gradients, but not very meaningful. An offset in elevation angle places the real structures in trace gas volume mixing ratios at slightly shifted altitudes. The actual volume mixing ratios in the structures are, thereby, not largely affected (at least for the offsets potentially present). In a conventional error estimate, a slight shift of a peak in volume mixing ratio, therefore, results in a very large absolute error in the vicinity of this structure.

To capture the influence of this error in a more practical manner, we perform retrievals with an elevation angle offset increased and decreased by $0.02^{\circ}$. These derive structures with a slightly different altitude structure. We then map the shifted structures back to their original location by removing the geometrical effect of the elevation angle offset from the retrievals. By calculating the difference in trace gas volume mixing ratios between these retrievals and the original one, we separate the effect of the elevation offset angle error on absolute values of trace gas volume mixing ratios from its effect on the vertical placement of structures. The effect on absolute values of volume mixing ratios is then incorporated into our error estimates while the effect on vertical trace gas structure placement is captured in Table 3.

The forward model error is estimated by comparing the radiances produced by our forward model for the retrieved atmospheres to calculations employing the RFM. The error varies over tangent altitude and spectral band and its standard deviation lies between $0.1 \%$ and $0.2 \%$ for different IMWs (see Table 1).
Our diagnostic system would also allow the creation and use of an accurate measurement error covariance matrix for the retrieval itself. However, we found that using such a matrix containing many systematic correlated errors causes our retrieval algorithm to accept solutions with systematic discrepancies of more than $30 \%$ between simulated and measured radiances. Thus, as stated above, we assume an uncorrelated error covariance matrix $\mathbf{S}_{\epsilon}$ with an assumed error of $1 \%$, as was already used by Weigel et al. (2010).

We calculate the error with respect to all non-retrieved gases, instrument errors (gain, offset, elevation angle offset), forward model error and spectroscopic line data uncertainties. These errors are summed up quadratically and the square root of the result is referred to as "total" error. Even though we do not employ a real covariance matrix for the a priori state, we find it useful to include the smoothing error $\mathbf{S}_{\mathrm{s}}=(\mathbf{A}-\mathbf{I}) \mathbf{S}_{\mathrm{a}}(\mathbf{A}-\mathbf{I})^{\mathrm{T}}$, as this only grows to significant proportions in regions where insufficient measurement information is present and, thereby, highlights this defect. The error term including both the total and the smoothing error is referred to as "total + smooth" and is used below for error estimates and error bars (except for Fig. 14).

\section{Registration of profiles}

The elevation angle of the measured spectra is an important quantity when evaluating limb sounder measurements. The elevation angle of a vertical scan with respect to the instrument reference system is fairly well known from the CRISTA-NF attitude system. However, external absolute attitude information (such as that provided by the MIPAS-STR attitude system) is needed to transfer these values into absolute elevation angles with respect to the Earth. Due to drifts of the CRISTA-NF gyro measurements and insufficient accelerometer measurements, absolute attitude information are not available from the CRISTA-NF attitude system.

In the attitude correction step, one elevation angle offset per flight (arising from the slightly different orientations of the attitude systems of MIPAS-STR and CRISTA-NF) is derived from roll and pitch angle measurements taken during a time span with few aircraft movements and vibrations (before take-off or after landing). For some flights, this is difficult, as the time span where both instruments recorded valid attitude measurements is very short. For other flights, no such measurements are available. To validate the elevation angle offset derived in this manner and to evaluate flights for which no quiet time period can be evaluated, the reference elevation angle offset must also be retrieved from the observed spectra.

Estimating this basic elevation angle offset during the retrieval is not trivial, as it is a very nonlinear parameter and the retrieval often accommodated other retrieved quantities (especially temperature) to the initial guess used for the elevation angle offset. Instead, we perform a series of flight retrievals with different fixed elevation angle offsets and 

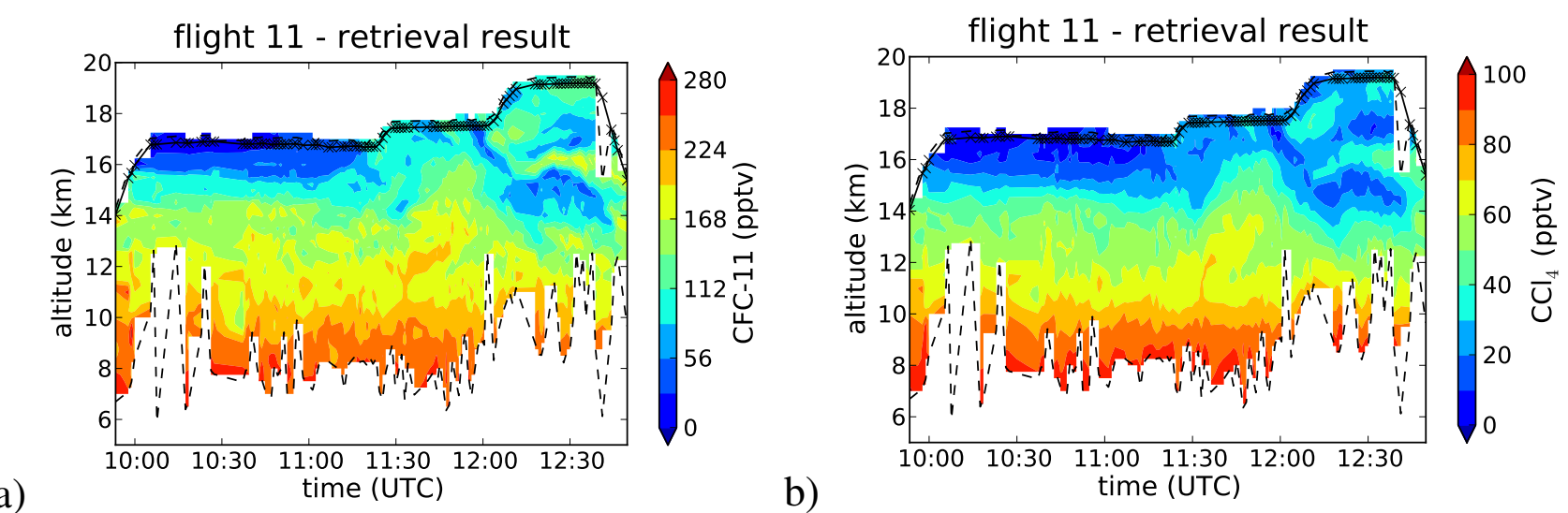

b)

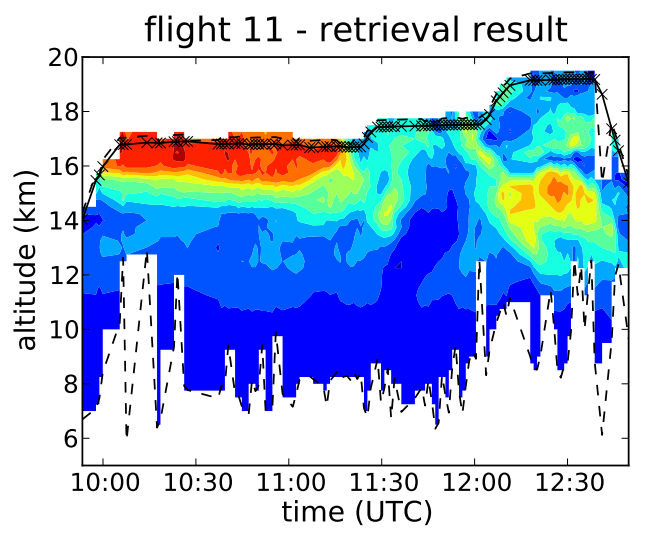

c)

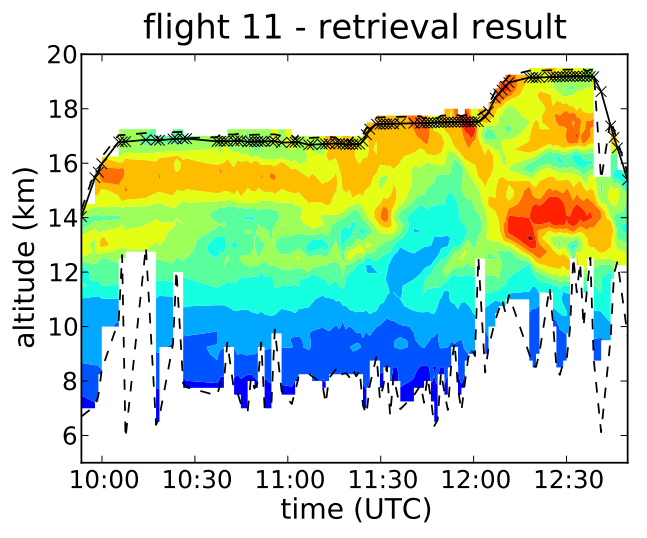

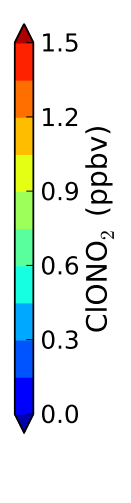

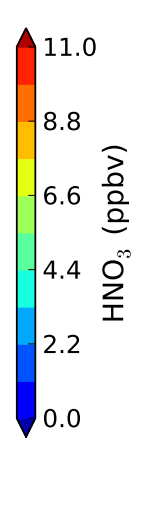

d)
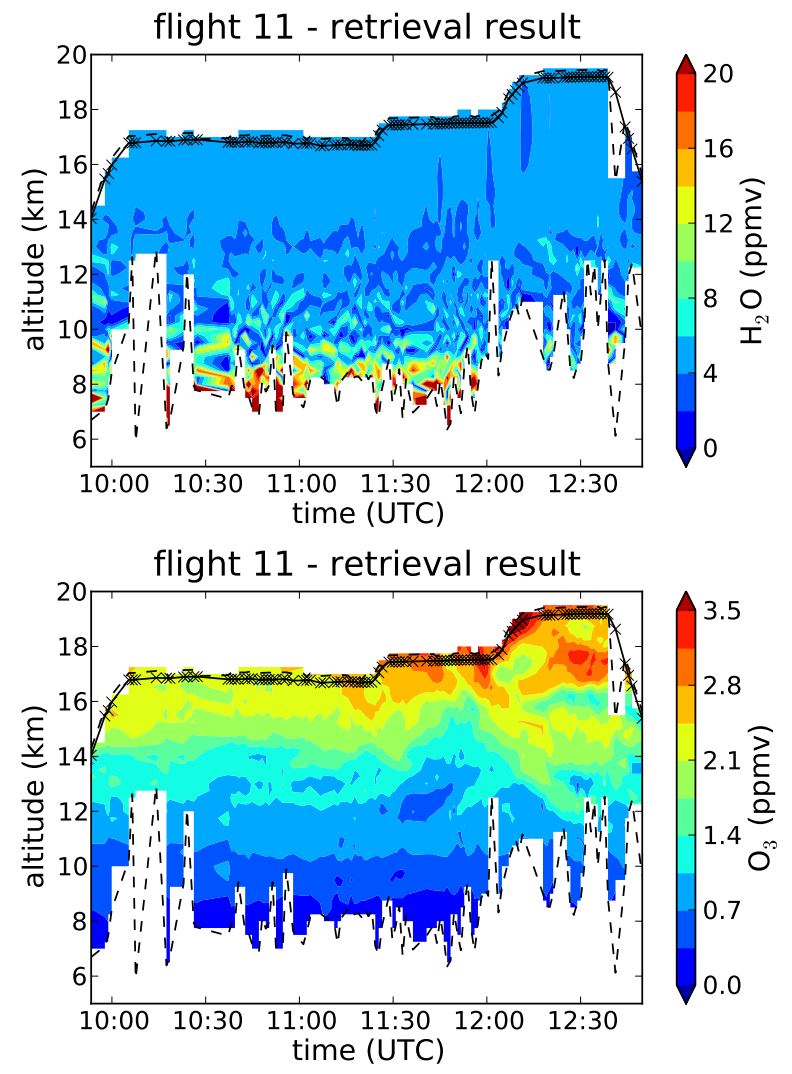

Fig. 5. Derived mixing ratio cross-sections for $\mathrm{CCl}_{4}$ and $\mathrm{CFC}-11, \mathrm{ClONO}_{2}, \mathrm{H}_{2} \mathrm{O}, \mathrm{HNO}_{3}$ and $\mathrm{O}_{3}$. The time, at which the profile was taken, is shown on the horizontal axis while the altitude of retrieved atmospheric quantities determines the vertical axis. The straight line indicates the observer altitude with crosses marking the position of measured profiles. Dashed lines indicate the altitude of highest and lowest valid measurement.

compare the average of the fit of measurements to the simulated measurements for the retrieval result over all vertical scans of one flight. To focus on the $\mathrm{CO}_{2}$ emissions, we fix temperature to the values supplied by ECMWF. Even though any bias in ECMWF temperatures might affect the derived elevation angle, this bias is expected to be small as we take into account all profiles of one flight representing a large volume of air. Table 4 shows the average fit over all profiles of one flight of the actual measurements to simulated measure- ments $\left(\xi_{\mathrm{m}}^{2}\right)$ for a series of different elevation angle offsets. There is a minimum around $0.00^{\circ}$ for this flight, indicating that the elevation angles derived by the calibration process are correct. As the minimum is not very sharp, we assume an ad hoc standard deviation for the elevation angle offset of $0.02^{\circ}$. 
Table 3. Vertical shift of retrieved data for different elevation angle errors and altitudes below the observer.

\begin{tabular}{ccccc}
\hline vert. distance & $-0.04^{\circ}$ & $-0.02^{\circ}$ & $0.02^{\circ}$ & $0.04^{\circ}$ \\
\hline $2 \mathrm{~km}$ & $-113 \mathrm{~m}$ & $-56 \mathrm{~m}$ & $55 \mathrm{~m}$ & $110 \mathrm{~m}$ \\
$4 \mathrm{~km}$ & $-159 \mathrm{~m}$ & $-79 \mathrm{~m}$ & $78 \mathrm{~m}$ & $156 \mathrm{~m}$ \\
$6 \mathrm{~km}$ & $-195 \mathrm{~m}$ & $-97 \mathrm{~m}$ & $96 \mathrm{~m}$ & $192 \mathrm{~m}$ \\
$8 \mathrm{~km}$ & $-225 \mathrm{~m}$ & $-112 \mathrm{~m}$ & $111 \mathrm{~m}$ & $221 \mathrm{~m}$ \\
$10 \mathrm{~km}$ & $-251 \mathrm{~m}$ & $-125 \mathrm{~m}$ & $124 \mathrm{~m}$ & $248 \mathrm{~m}$ \\
\hline
\end{tabular}

\section{Results for flight 11}

This section presents the results for the 11th RECONCILE flight that took place on 2 March 2010 between 09:34 and 13:35 UTC, hereafter called flight 11 . Only a few of the derived quantities are shown in plots below. Only the six primary retrieval targets $\mathrm{CCl}_{4}$ and $\mathrm{CFC}-11, \mathrm{ClONO}_{2}, \mathrm{H}_{2} \mathrm{O}$, $\mathrm{HNO}_{3}$ and $\mathrm{O}_{3}$ will be discussed in detail. The secondary retrieval targets are only retrieved to reduce the error for the primary retrieval targets. They behave similarly to the primary retrieval targets, but typically with a decreased resolution and/or measurement content. We would like to note that the resulting profiles for CFC-113 and HCFC-22 deliver volume mixing ratios that are roughly consistent with polar winter profiles derived by the Atmospheric Chemistry Experiment Fourier Transform Spectrometer (ACE-FTS; Bernath et al., 2005).

The flight path of the M55-Geophysica during RECONCILE flight 11 is depicted in Fig. 4. The plane started from Spitsbergen and first flew North. It then turned South and flew straight towards the airport of Kiruna. Flight locations, from which profiles were measured by CRISTA-NF, are marked by crosses. The tangent point locations of such profiles are shown as circles with colour-coding indicating the geometric altitude of the tangent point. Two profiles are highlighted and will be referenced to below. The profile taken at 11:07 UTC coincides roughly with the air mass traversed by the aircraft during its ascent. It is also representative of the first part of the flight inside the remnant of the polar vortex with the CRISTA-NF measurements covering air inside and below the polar vortex. The other highlighted profile was selected as representative of the second part of the flight outside this region. Only a subset of retrieved profiles is presented. To filter out profiles with obvious defects, e.g., due to stronger movements by the aircraft than the attitude system can compensate for, only profiles where the term describing the fit of the profile to the measurements in the cost function could be reduced to less than 0.65 are used (i.e., $\left.\left(\boldsymbol{F}\left(\boldsymbol{x}_{\mathrm{f}}\right)-\boldsymbol{y}\right)^{\mathrm{T}} \mathbf{S}_{\epsilon}^{-1}\left(\boldsymbol{F}\left(\boldsymbol{x}_{\mathrm{f}}\right)-\boldsymbol{y}\right)<0.65\right)$. This value was chosen in an ad-hoc manner to consistently filter out profiles with obvious defects.
Table 4. Fit of measurements for cross-section retrievals with different fixed elevation angle offsets.

\begin{tabular}{cc}
\hline elevation angle $\left(^{\circ}\right)$ & $\xi_{\mathrm{m}}^{2}$ \\
\hline-0.10 & 0.992 \\
-0.03 & 0.442 \\
-0.02 & 0.416 \\
-0.01 & 0.380 \\
-0.00 & 0.369 \\
-0.01 & 0.372 \\
-0.02 & 0.392 \\
-0.03 & 0.396 \\
-0.10 & 0.396 \\
\hline
\end{tabular}

\subsection{Derived trace gas volume mixing ratios}

In this section, derived trace gas volume mixing ratios and the associated diagnostic quantities are presented and discussed.

Figure 5 shows six cross-sections with derived trace gas volume mixing ratios. The plots are assembled from a series of retrieved profiles. Only atmospheric quantities located between the dashed lines are plotted, as only these are sufficiently determined by measurements in contrast to being more determined by a priori information.

Figure 5a shows CFC-11 volume mixing ratios. CFC-11 volume mixing ratios typically fall with altitude and low volume mixing ratios indicate usually stratospheric air. The very low CFC-11 volume mixing ratios to the upper left above $15 \mathrm{~km}$ probably show the extent of the polar vortex (compare also with potential vorticity distribution in Fig. 4), whereas the low volume mixing ratios to the lower right are likely remnant of polar vortex air. The air mass with low volume mixing ratios on the upper right could be of a different origin as the ozone volume mixing ratios in this filament seem to be too high to be a recent remnant of vortex air. Please note the thin filament of increased CFC-11 volume mixing ratio at $\approx 16 \mathrm{~km}$ on the right, probably originating from midlatitudes. This thin filament has a vertical extent of 0.5 to $1 \mathrm{~km}$ and its signature can be found in all primary target species except water vapour.

The distribution of $\mathrm{CCl}_{4}$ in Fig. $5 \mathrm{~b}$ is very similar to the distribution of CFC-11, but fewer filaments are visible due to the decreased vertical resolution compared to CFC-11.

$\mathrm{ClONO}_{2}$ is a chlorine reservoir species found in abundance in the stratosphere. The $\mathrm{ClONO}_{2}$ volume mixing ratios depicted in Fig. 5c show largely high volume mixing ratios where low CFC-11 volume mixing ratios are given and vice versa. This is expected from their climatological volume mixing ratios, which decrease in altitude for CFC-11 and increase for $\mathrm{ClONO}_{2}$ in the presented altitude range.

Figure $5 \mathrm{~d}$ shows that the water vapour content in this polar atmosphere is roughly $5 \mathrm{ppmv}$, which is consistent with climatological profiles. The colour scale for the water vapour 
a)
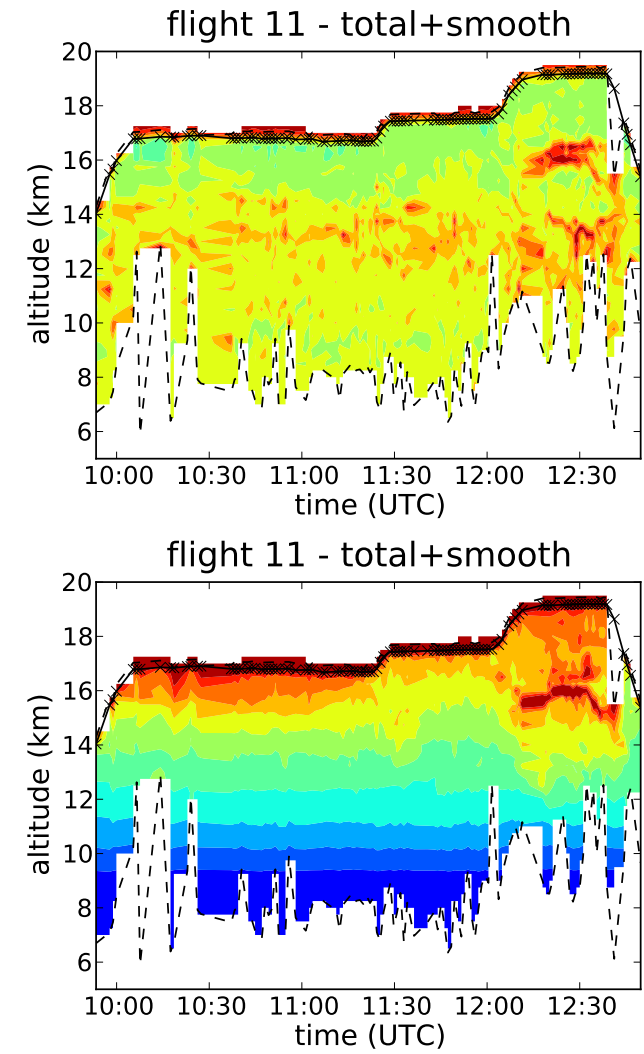

c)

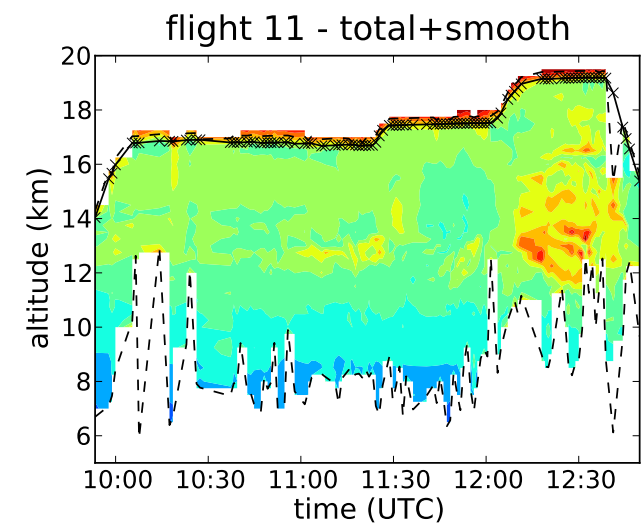

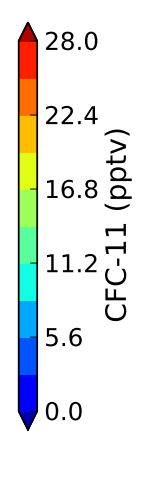

b)
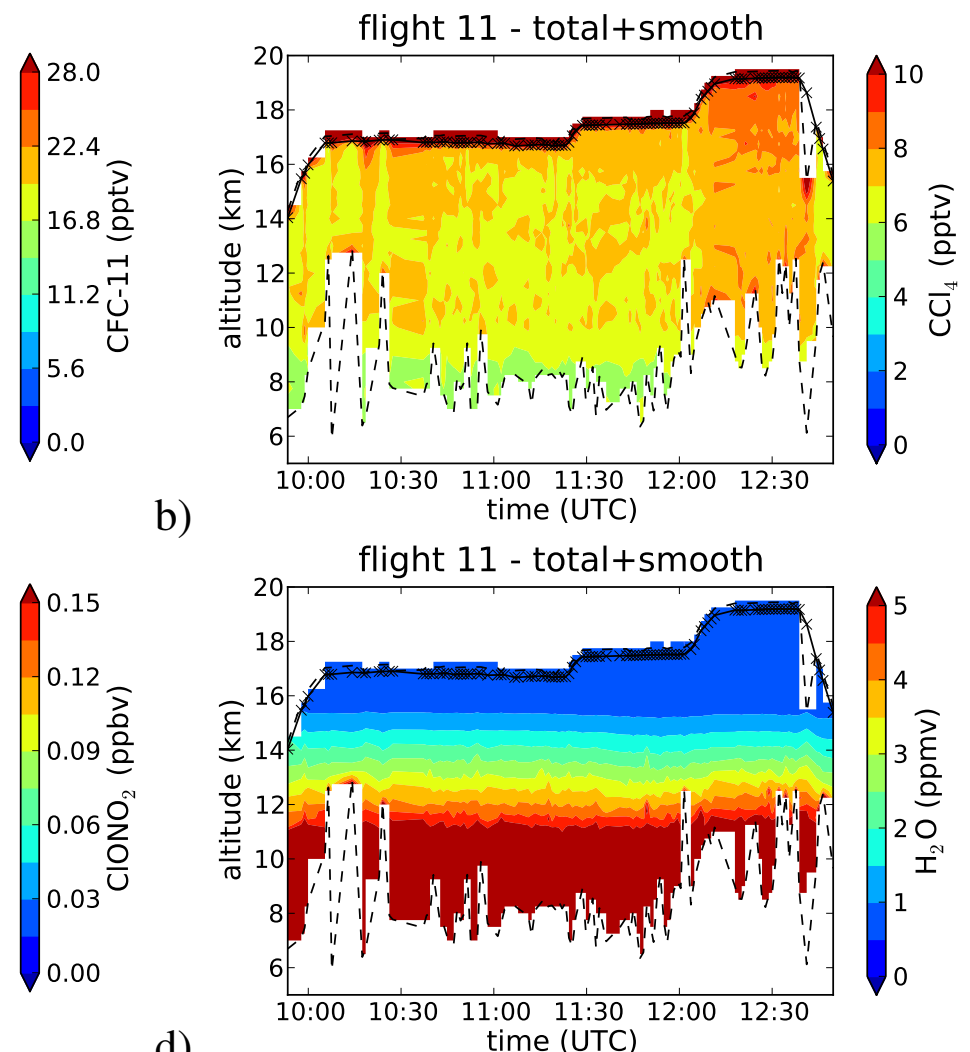

d)
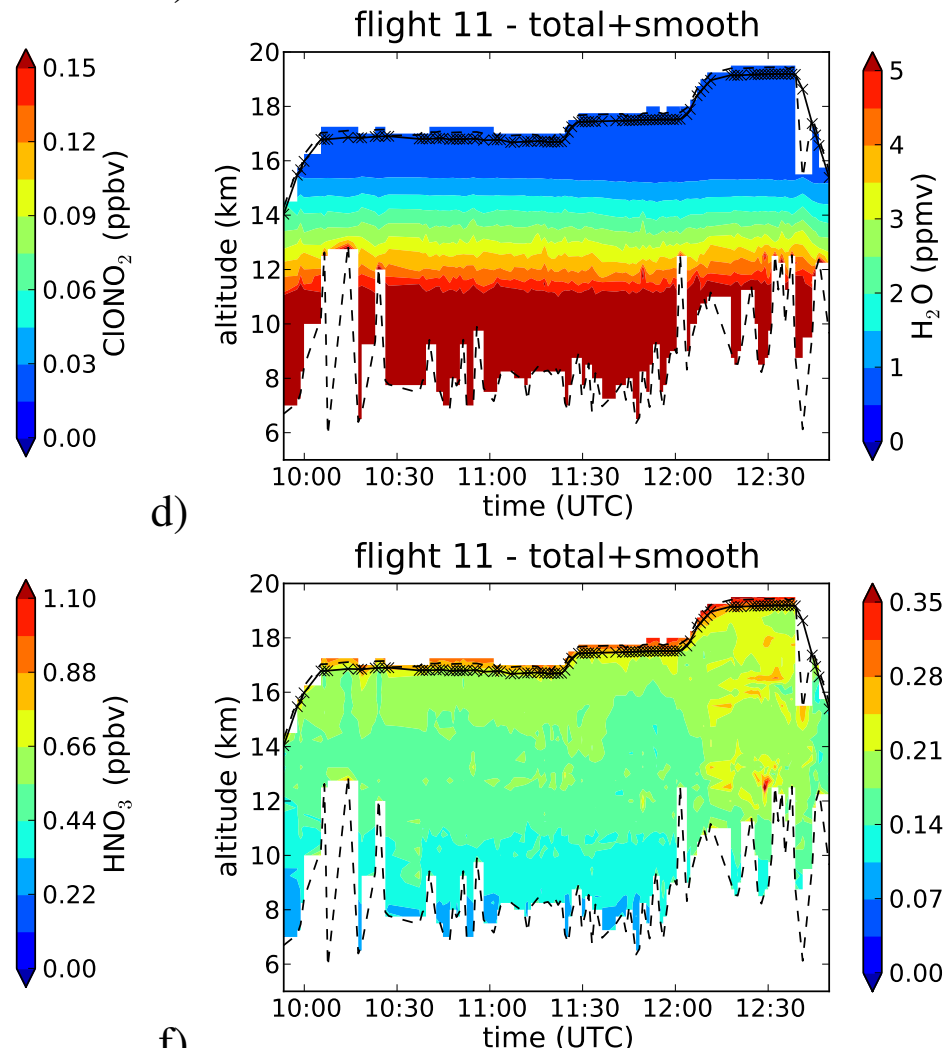

f)

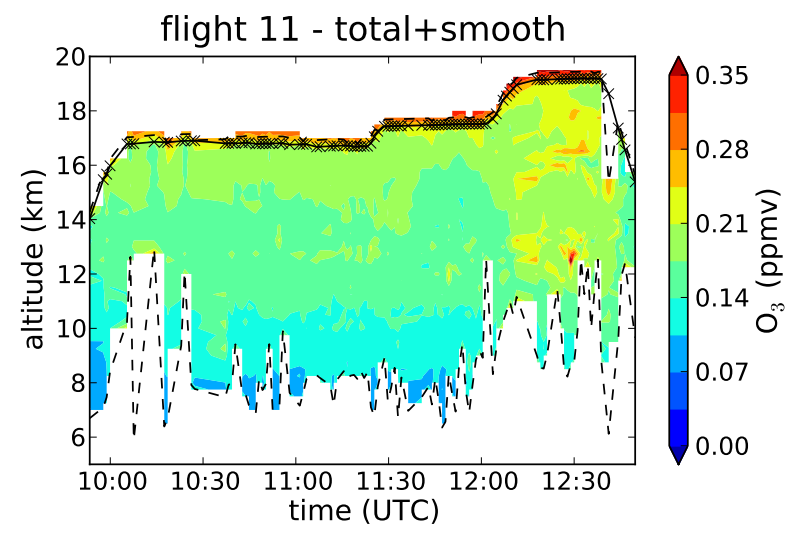

Fig. 6. "Total + smooth" errors for $\mathrm{CCl}_{4}$ and $\mathrm{CFC}-11, \mathrm{ClONO}_{2}, \mathrm{H}_{2} \mathrm{O}, \mathrm{HNO}_{3}$ and $\mathrm{O}_{3}$.

graph was chosen to be consistent with our error bar, implying that we cannot significantly resolve more details than depicted.

The volume mixing ratios of the stratospheric trace gas $\mathrm{HNO}_{3}$ are shown in Fig. 5e. Please note the fine structures in $\mathrm{HNO}_{3}$ volume mixing ratios, especially the horizontal filaments of increased volume mixing ratio of about $6 \mathrm{ppb}$ at $12 \mathrm{~km}$ and $13.5 \mathrm{~km}$ in the middle part of the figure as well as the structure at $12 \mathrm{~km}$ to the right, where air with increased $\mathrm{HNO}_{3}$ volume mixing ratio apparently surrounds a filament with decreased volume mixing ratio.
Figure $5 \mathrm{f}$ depicts the volume mixing ratios for $\mathrm{O}_{3}$. Its distribution is similar to the other stratospheric trace gases. The comparatively low volume mixing ratios to the upper left and lower right may indicate air with depleted ozone most likely stemming from the polar vortex.

An in-depth discussion about the atmospheric situation can be found in Kalicinsky et al. (2012).

The associated errors are shown in Fig. 6. Due to the uncertainty in elevation angle, the error becomes especially large in the vicinity of large vertical gradients, as can be seen most easily in Fig. 5a around 12:30. Due to the uncertainty 
a)

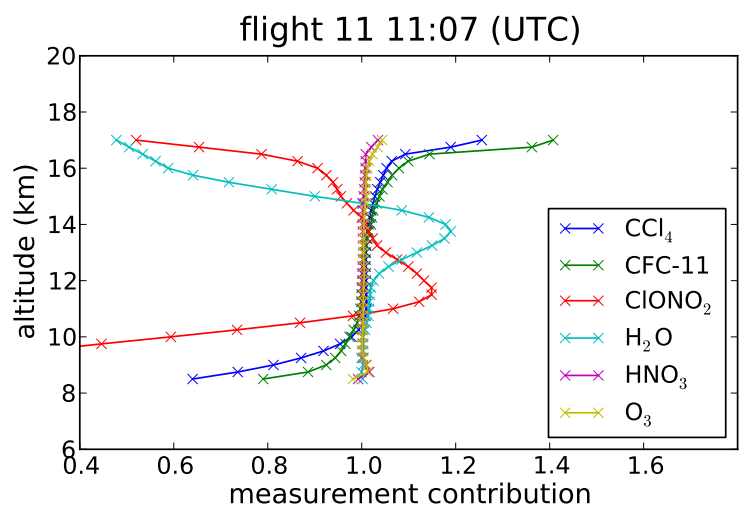

b)

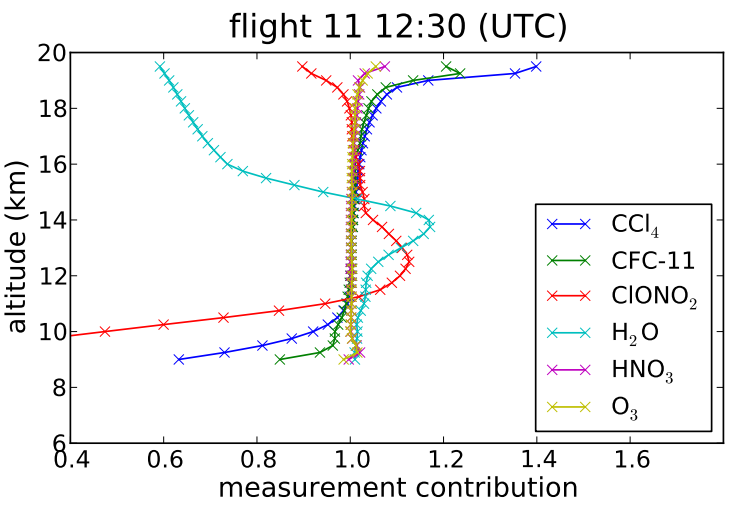

Fig. 7. Measurement contribution (sum of rows of averaging kernel matrix) for $\mathrm{CCl}_{4}$ and $\mathrm{CFC}-11, \mathrm{ClONO}_{2}, \mathrm{H}_{2} \mathrm{O}, \mathrm{HNO}_{3}$ and $\mathrm{O}_{3}$ for profiles at 11:07 in (a) and 12:30 UTC in (b).

in position of the thin CFC-11 filament, the relative error is increased to more than $10 \%$.

The measurement contribution for the two profiles highlighted in Fig. 4 is shown in Fig. 7. Again, only data points in between the highest and lowest tangent point are depicted. These two profiles were selected as typical of the first part of the flight inside the polar vortex (11:07 UTC in Fig. 5a) and for the second part of the flight above the large remnant of vortex air (12:30 UTC in Fig. 5b). The unit-less measurement contribution is close to 1 within the height range covered by measurements. Towards lower altitudes, the measurement contribution becomes smaller as denser air and increasing cross-talk reduce the signal strength. Going down from top to bottom, the measurement contribution for water vapour is first very small as the signal of these low water vapour mixing ratios is below the detection limit in the spectral range used for retrieval. It then increases above 1 and then drops back to 1 and stays there down to the lowest valid data points. Similarly, but in opposite direction, going again from top to bottom, the measurement contribution of $\mathrm{ClONO}_{2}$ is first close to one, then increases and finally drops towards the surface due to a lack of signal. We hypothesize the increase above 1 to be an artefact of the low trace gas volume mixing ratio, which is below the detection limit of the

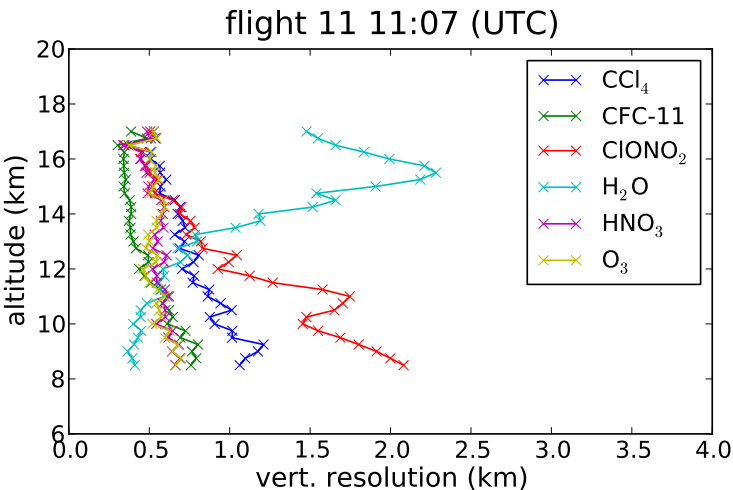

a)

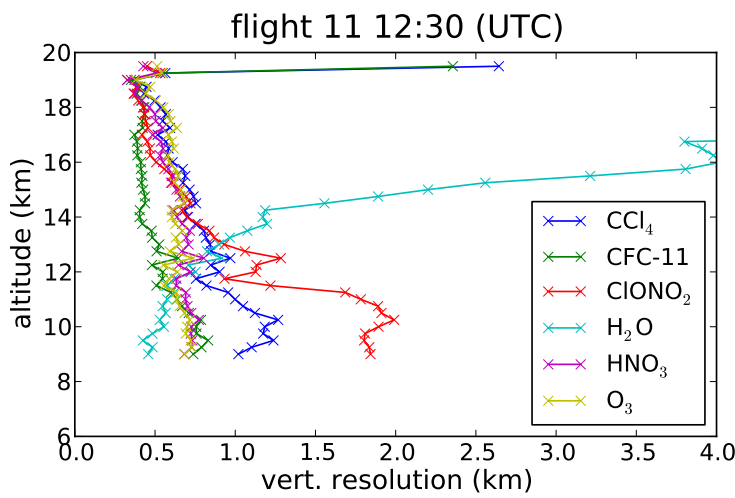

b)

Fig. 8. Vertical resolution for $\mathrm{CCl}_{4}$ and $\mathrm{CFC}-11, \mathrm{ClONO}_{2}, \mathrm{H}_{2} \mathrm{O}$, $\mathrm{HNO}_{3}$ and $\mathrm{O}_{3}$ for profiles at 11:07 in (a) and 12:30 UTC in (b).

IMWs employed here. The bump above 1 can be removed, e.g., by not retrieving $\mathrm{ClONO}_{2}$ in the lowest $10 \mathrm{~km}$ of the profile, but assuming the a priori values instead; this delivers nearly identical $\mathrm{ClONO}_{2}$ volume mixing ratios above $10 \mathrm{~km}$ with the measurement contribution staying close to 1 . However, the abrupt change between retrieved $\mathrm{ClONO}_{2}$ volume mixing ratios and values fixed to the a priori in the lower part of the profile causes other undesirable artefacts in retrieved volume mixing ratios and diagnostics, so it was not applied to the presented results. The increased measurement contribution in water vapour could be treated similarly by not retrieving water vapour volume mixing ratios above $12 \mathrm{~km}$, as this removes the oversensitivity. However, we do not see the increased measurement contribution as a problem as the retrieved volume mixing ratios in the affected altitude range are close to the detection limit and, therefore, of low quality to begin with.

The vertical resolution of the same two profiles is depicted in Fig. 8. The irregular structure of the resolution values stems from the discrepancies between the measurement grid and the retrieval grid, on the one hand, and the presence or absence of strong vertical gradients on the other. Except for water vapour, the vertical resolution generally starts below $500 \mathrm{~m}$ at the observer altitude and worsens with decreasing altitude. Especially CFC-11 is well resolved, with a vertical resolution of $\approx 400 \mathrm{~m}$ for $\approx 2 \mathrm{~km}$ below the aircraft. Such 
a high vertical resolution is exceptional for a limb sounder instrument. These results show that increasing the vertical sampling density of a limb sounder to the given granularity still improves the quality of the retrieval results. The feasibility of limb observations with such a high vertical resolution is also a valuable finding for the proposed satellite-based limb-imaging technique (Riese et al., 2005; ESA, 2008) that will be characterised by a combination of high vertical sampling (order of $500 \mathrm{~m}$ ) and a comparatively small vertical FOV. For CFC-11, ozone, and $\mathrm{HNO}_{3}$, the vertical resolution stays well below $1 \mathrm{~km}$ over the whole relevant altitude range, while $\mathrm{CCl}_{4}$ and $\mathrm{ClONO}_{2}$ become worse. No IMW without strongly interfering species is available for the latter gases, so they are more severely affected by loss of signal at lower altitudes. There, the transmissivity of most of the IMWs drops down to $0.2-0.5$ from about 0.9 at observer altitude, which together with the increasing FOV explains the decrease in resolution. The resolution of water vapour similarly worsens towards higher altitudes as the signal to noise ratio of the employed IMWs is insufficient for the water vapour volume mixing ratios present. Please note the strong connection between the drop in measurement contribution of $\mathrm{ClONO}_{2}$ and water vapour and the worsening vertical resolution. Simulations for the air-borne GLORIA instrument by Ungermann et al. (2010) indicate that imager instruments with an even higher vertical sampling (order of $125 \mathrm{~m}$ ) than offered by CRISTA-NF can attain vertical resolutions down to 250 to $300 \mathrm{~m}$.

The horizontal resolution along the LOS in Fig. 9 gives insight into the potential effect of horizontal gradients along the LOS on derived trace gas volume mixing ratios. For the same profiles, the diameter of the sphere containing all the elements of the averaging kernel matrix equal to or larger than half of the maximum element of each row is depicted. The horizontal resolution along the LOS starts with about $100 \mathrm{~km}$ at the observer altitude and increases rapidly in both cases to $300-400 \mathrm{~km}$. Towards lower altitudes, the increase slows down, as the IMWs become more and more optically dense and the maxima of the weighting functions become increasingly shifted towards the observer.

\subsection{Validation}

This section compares retrieved trace gas volume mixing ratios with the corresponding values measured by other instruments. The M55-Geophysica is capable of carrying many different scientific instruments. During the RECONCILE campaign, more than 20 different remote sensing and in situ instruments were mounted on the aircraft. This offers the unique opportunity to compare the results of our CRISTANF study with the measurements of different instruments. On the one hand, there are in situ instruments, of which two also measure primary retrieval targets of ours (CFC-11 and ozone, see below). On the other hand, there is the passive infrared limb-sounder MIPAS-STR, which has been exten-

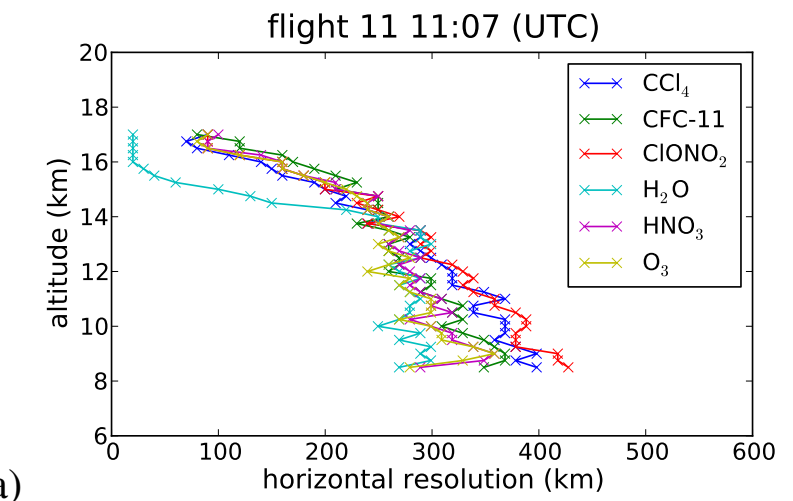

a)

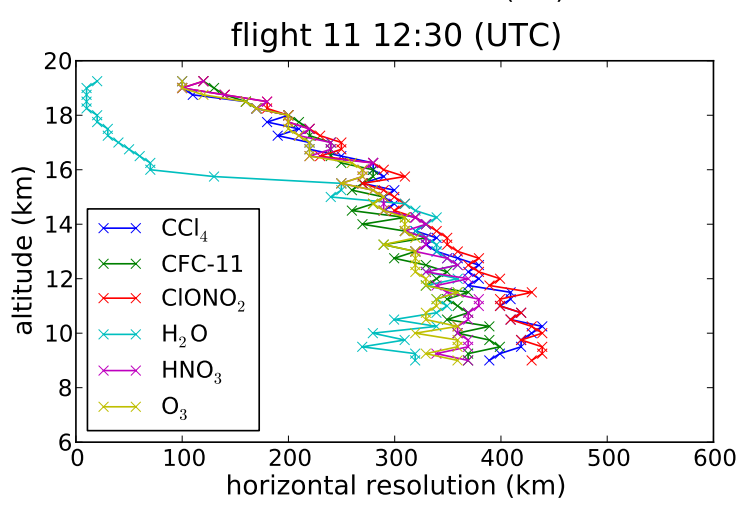

b)

Fig. 9. Horizontal resolution along $\mathrm{LOS}$ for $\mathrm{CCl}_{4}$ and CFC-11, $\mathrm{ClONO}_{2}, \mathrm{H}_{2} \mathrm{O}, \mathrm{HNO}_{3}$ and $\mathrm{O}_{3}$ for profiles at 11:07 in (a) and 12:30 UTC in (b).

sively validated in the past and can, therefore, also be seen as a reference, taking into account the different capabilities with respect to spatial and spectral resolution.

While we cannot validate all our primary retrieval targets, showing the consistency of CFC-11 and especially ozone with in situ measurements provides confidence in the results for other targets.

\subsubsection{Comparison with HAGAR}

The first comparison shows CFC-11 volume mixing ratios measured by the in situ High Altitude Gas AnalyzeR (HAGAR; Riediger et al., 2000; Werner et al., 2010) plotted against the retrieved CFC-11 quantities at the observer altitude. Figure 10 shows volume mixing ratios retrieved from CRISTA-NF measurements as a blue line with error bars indicating the "total + smooth" error and HAGAR volume mixing ratios as a green line with crosses indicating the time of measurements. Errors of the HAGAR measurements are smaller than the green symbols. The observer altitude is shown as a dotted black line with a second axis to the right. The CFC-11 volume mixing ratios overall agree with one another, mostly even within the one sigma indicated by the error bar. Remaining discrepancies can be explained by the viewing geometry and spatial averaging characteristics of the limb 


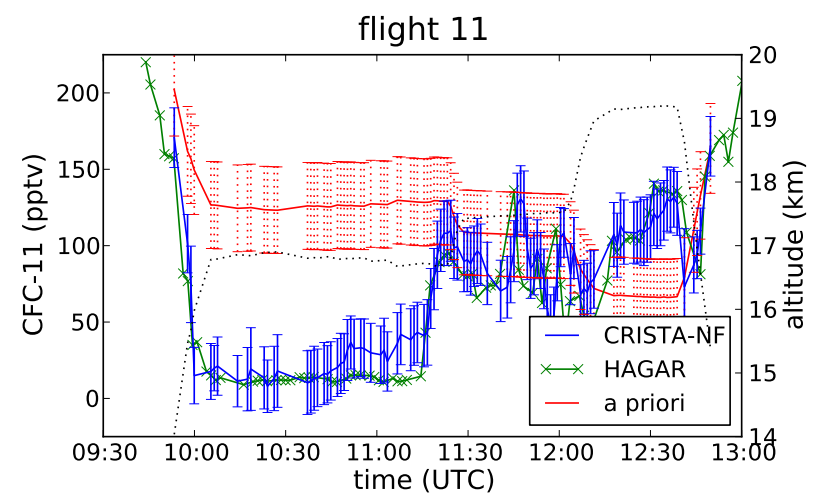

Fig. 10. Comparison of CFC-11 mixing ratios as measured by HAGAR and derived by CRISTA-NF. Shown in blue are CRISTANF results including error bars showing the "total + smooth" error. Shown in green are HAGAR measurements. Shown in red is the a priori. The dotted line indicates the plane altitude at measurement time with scale on the right.

sounder compared to the in situ sampling by HAGAR. For example, the increase in CRISTA-NF CFC-11 volume mixing ratio from 10:40 to 11:15 UTC precedes the corresponding increase of the HAGAR CFC-11 volume mixing ratio. While the in situ instrument presumably samples air from the remnant of the polar vortex, the CRISTA-NF instrument also sees air from outside the vortex, which contains larger $\mathrm{CFC}-11$ volume mixing ratios. This assumption is supported by the distribution of potential vorticity shown as contours in Fig. 4. At this time, the plane is located in airmasses with a potential vorticity above $26 \mathrm{PVU}$ and the instrument looks towards airmasses with lower potential vorticity. Further, the slight time shift in the detection time of filaments of high CFC-11 volume mixing rations at 11:45 and 11:58 UTC is also explainable by the viewing geometry. The distribution of potential vorticity suggests that the filaments are slightly slanted with respect to the viewing geometry with the aircraft entering the filament first before it comes into full view of the instrument. A second comparison is shown in Fig. 11. The M55-Geophysica ascended from the ground to flight altitude between 09:34 and 10:04 UTC. During this time frame, the in situ instruments including HAGAR already collected data. We assembled the ascent measurements by HAGAR in Fig. 11 with green crosses indicating data points. CRISTANF measured roughly the same air mass between 10:55 and 11:20 UTC. We interpolate our retrieved profiles to approximately match the air masses measured by the in situ instrument. The CFC-11 profile derived from this interpolation is plotted in blue including error bars. While the more sparsely sampled HAGAR profile does not show the same structures as the CRISTA-NF profile, they agree well with respect to average absolute values.

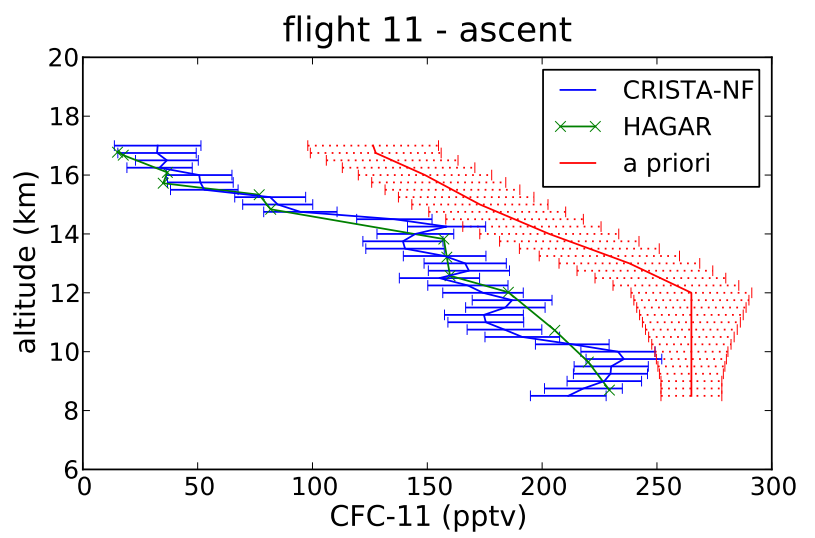

Fig. 11. Comparison of CFC-11 mixing ratios as measured by HAGAR and derived by CRISTA-NF. HAGAR measurements are taken from the ascent of the plane from 09:34 to 10:04 UTC while the CRISTA-NF profile is assembled from several retrieved profiles taken between 10:55 and 11:20 UTC that measure roughly the same air mass.

\subsubsection{Comparison with FOZAN}

The second comparison proceeds in a similar manner to the first, but for ozone volume mixing ratios as measured by the Chemiluminescent Fast Ozone Analyzer (FOZAN; Ulanovsky et al., 2001). Figure 12 shows again the volume mixing ratios at observer altitude, which largely agree within error bars. Especially during the first half of the flight, where the ozone volume mixing ratios are very stable, the values of the instruments are very close, with typical slightly lower values for CRISTA-NF. The underestimation in the ozone at observer altitude seems correlated to overestimation in CFC-11, which indicates that this might be the effect of horizontal gradients along the LOS. Peaks and troughs in ozone volume mixing ratio as measured by FOZAN between 11:15 and 12:15 UTC are also measured by CRISTA-NF, but with a time delay. This might indicate that these structures stem from filaments that are not completely orthogonal to the flight path as is supported by the distribution of potential vorticity at $17 \mathrm{~km}$ in Fig. 4.

The ascent measurements can be compared in the same way as described in the preceding section and the comparison is shown in Fig. 13. The measured values agree very well with one another, mostly within much less than one sigma of the CRISTA-NF error. Even though the CRISTA-NF instrument has a much poorer vertical resolution and even in the presence of a time-shift of more than one hour, there is a remarkable agreement in the fine structure. For example, the decrease in ozone at $13.5 \mathrm{~km}$ is captured just as the increase at $14.0 \mathrm{~km}$.

The agreement between FOZAN and CRISTA-NF is much better for the ascent profile than for the comparison along the flight track. The air measured by FOZAN during the ascent of the M55-Geophysica lies well within the region 


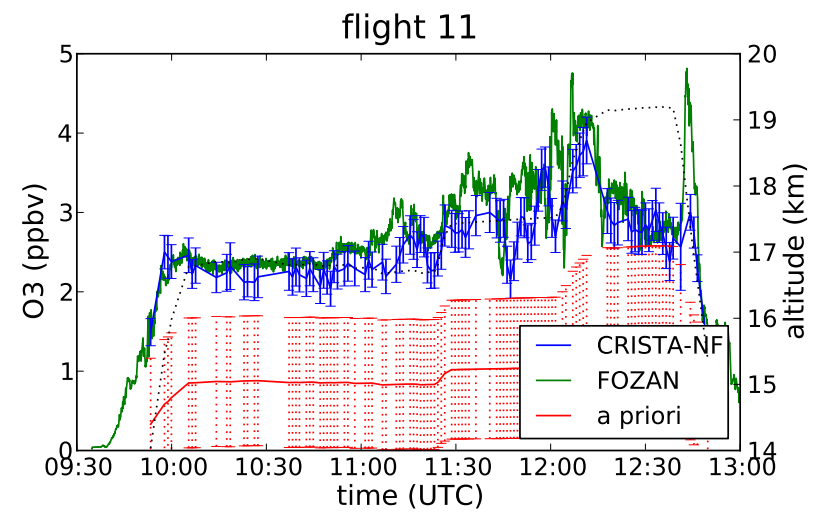

Fig. 12. Comparison of ozone mixing ratios as measured by FOZAN and derived by CRISTA-NF. Shown in blue are CRISTANF results including error bars showing the "total + smooth" error. Shown in green are FOZAN measurements. Shown in red is the a priori. The dotted line indicates the plane altitude at measurement time with scale on the right.

from which most radiation is received by CRISTA-NF, so that a better fit is expected. Please note also that the largest horizontal gradients in ozone are expected in the altitude region above roughly $16 \mathrm{~km}$ in the vicinity of the polar vortex due to ozone depletion in vortex airmasses. Further, the agreements on flight level is better for the northward part of the flight, where the potential vorticity suggests more similar air masses along the LOS. This suggests that the discrepancy in ozone volume mixing ratios at the observer position evident in Fig. 12 is at least partially an artefact of the spatial averaging characteristics of the remote-sensing measurement method.

\subsubsection{Comparison with MIPAS-STR}

This section compares one exemplary profile measured by CRISTA-NF against a profile as measured by the MIPASSTR instrument (Piesch et al., 1996). Comparisons for other profiles show qualitatively similar agreement, depending somewhat on how structured the atmosphere is. A detailed description of the MIPAS-STR retrieval for this campaign is given by Woiwode et al. (2011); this paper also presents 2-D cross-sections of $\mathrm{HNO}_{3}$ volume mixing ratios from both instruments, which underline the general extremely good level of agreement. For the profile-based comparisons shown here, a profile retrieved from a vertical scan taken at 12:30 in the latter part of the flight was selected as it contains the greatest vertical variability and the most complex structure. The volume mixing ratios of most primary species derived by CRISTA-NF (except for $\mathrm{CCl}_{4}$ ) are also derived by MIPASSTR albeit at a decreased spatial resolution due to the different sampling strategy and FOV of MIPAS-STR. The measurement grid distance of MIPAS-STR is generally $1 \mathrm{~km}$ between observer altitude and $8 \mathrm{~km}$ altitude and $1.5 \mathrm{~km}$ below. In addition, comprehensive upwards sampling is performed

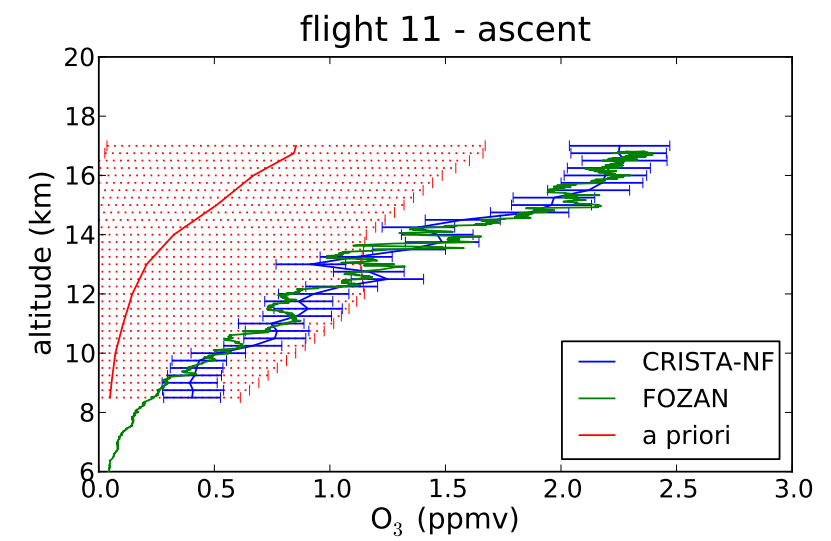

Fig. 13. Comparison of ozone mixing ratios as measured by FOZAN and derived by CRISTA-NF. FOZAN measurements are taken from the ascent of the plane from 09:34 to 10:04 UTC while the CRISTA-NF profile is assembled from several retrieved profiles taken between 10:55 and 11:20 UTC that measure roughly the same air mass.

in order to obtain limited vertical information on temperature and atmospheric constituents above the flight path. Depending on the sampling programme, the horizontal sampling density is between 25 and $45 \mathrm{~km}$. The FOV is $0.44^{\circ}$ (full cone). The MIPAS-STR retrieval grid is mainly finer than its FOV with a grid distance of $500 \mathrm{~m}$ in the relevant altitude range except for the uppermost observation geometries. Its resulting vertical resolution is largely 1 to $1.5 \mathrm{~km}$, which is about twice as much as the typical CRISTA-NF resolution. We mention that the error budget for the MIPASSTR retrieval results is generated differently compared to the CRISTA-NF error budget (e.g., conservative spectroscopic line data error, no smoothing error; compare Woiwode et al., 2011). For the MIPAS-STR retrievals, spectral line data was taken from the MIPAS database (see Flaud et al. (2006) and references cited therein), while for the CRISTA-NF retrievals HITRAN 2008 was applied, probably resulting in slightly different results.

Figure 14a shows side-by-side the ozone profiles retrieved by the two instruments including error bars (excluding the smoothing error for the CRISTA-NF results to be consistent with the supplied errors for MIPAS-STR) and the a priori values used for the CRISTA-NF retrieval. A very good agreement of absolute values is found above $13 \mathrm{~km}$, even though some of the dips in ozone volume mixing ratio do not seem to be fully resolved by MIPAS-STR. Taking into account the coarser resolution of MIPAS-STR, the profiles agree largely within the corresponding error bars.

The same is largely true for the CFC-11 profile shown in Fig. 14b. The fine filament of increased CFC-11 volume mixing ratios around $16 \mathrm{~km}$ altitude is also not fully resolved by MIPAS-STR; instead a lower peak is seen at a slightly increased altitude. However, the overall structures of the 
a)
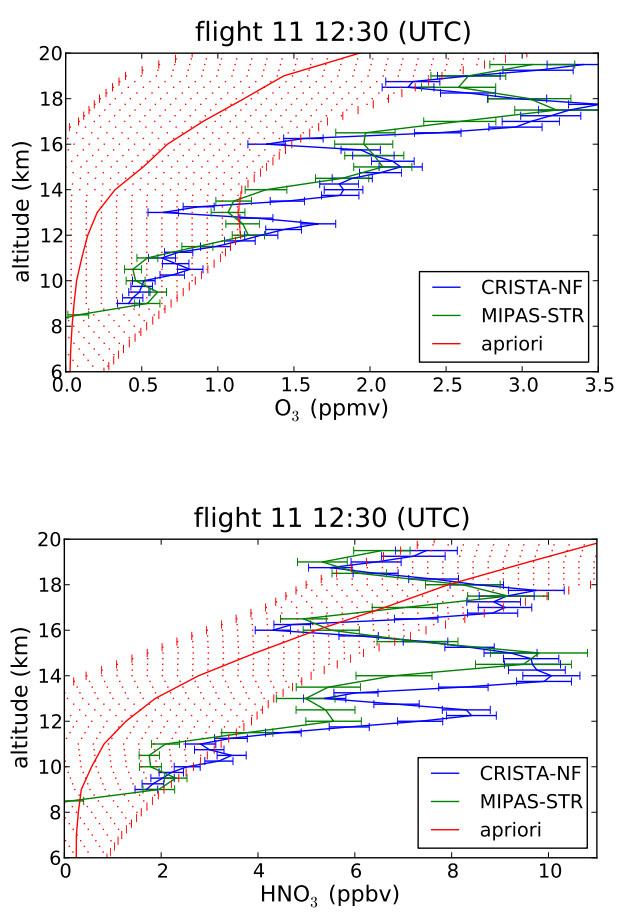

b)
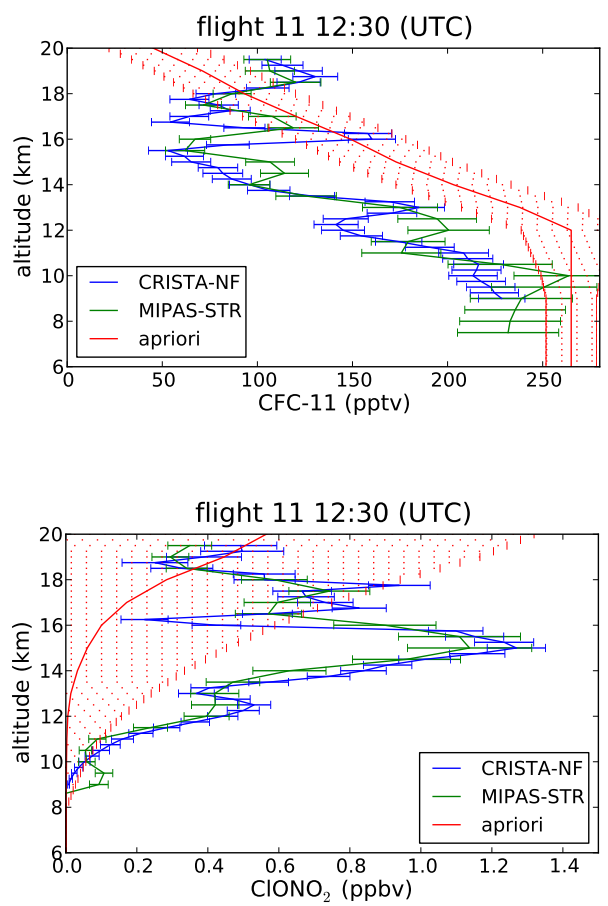

d)

flight 11 12:30 (UTC)

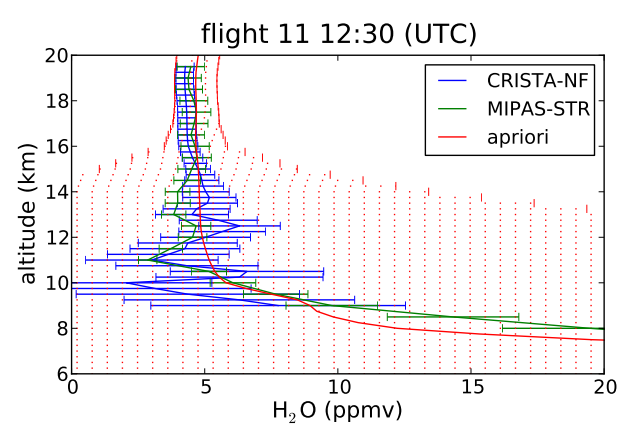

e)

Fig. 14. Comparison between retrieval results of CRISTA-NF (blue) and MIPAS-STR (green) for a single exemplary profile taken at 12:30 UTC during flight 11. The error bars show the "total error" only. Additionally shown in red is the a priori profile and standard deviations used for the CRISTA-NF retrievals.

profiles agree qualitatively and apart from the fine details related to filaments, the absolute values are also in agreement.

The $\mathrm{HNO}_{3}$ retrieval results are compared in Fig. 14c. Both instruments resolve three distinct peaks at $17 \mathrm{~km}, 14 \mathrm{~km}$ and $12 \mathrm{~km}$. CRISTA-NF generally sees slightly increased $\mathrm{HNO}_{3}$ volume mixing ratios compared to MIPAS-STR and also suggests a larger vertical extent of the $\mathrm{HNO}_{3}$ structures. This might indicate a systematic overestimation of this trace gas by CRISTA-NF. A comparison of the structures seen in the cross-sections is given by Woiwode et al. (2011).

Figure $14 \mathrm{~d}$ shows $\mathrm{ClONO}_{2}$ volume mixing ratios. There is a very good agreement between the two instruments. The maxima at $17 \mathrm{~km}$ and especially the structure around $15 \mathrm{~km}$ match very well. Only the dip in $\mathrm{ClONO}_{2}$ volume mixing ratio at $16 \mathrm{~km}$ cannot be fully resolved by MIPAS-STR. Any discrepancy is, however, consistent with the supplied error bars except for the dip at $16 \mathrm{~km}$.

The comparison in Fig. 14e shows the retrieved water vapour volume mixing ratios. Both instruments derive very low volume mixing ratios, which agree within error bars down to $9 \mathrm{~km}$ altitude.

\section{Summary and conclusions}

CRISTA-NF is a passive infrared limb sounder instrument, which was recently deployed during the RECONCILE campaign from January to March 2010. This paper discussed the evaluation process in general and especially the results and validation for flight 11 on 2 March 2010 . We briefly 
presented the process of calibrating the measurements taken during this campaign.

We employ a multi-target retrieval setup including 13 integrated microwindows to simultaneously derive volume mixing ratios of 9 trace gas species (primary targets: $\mathrm{CCl}_{4}$, CFC-11, $\mathrm{H}_{2} \mathrm{O}, \mathrm{HNO}_{3}, \mathrm{O}_{3}$ and $\mathrm{ClONO}_{2}$; secondary targets: PAN, CFC-113 and HCFC-22), temperature and aerosol. Our Tikhonov regularisation is modelled after the Bayesian auto-regressive model. It, thereby, delivers a simple altitudedependent regularisation, but remains easily tunable. Further, we demonstrated a simple way to estimate the horizontal resolution along the line-of-sight (LOS).

In the second part of the paper, we presented the retrieval results. We first showed cross-sections of trace gas volume mixing ratios, which show in great detail fine vertical and horizontal structures. The vertical resolution close to the aircraft is below $500 \mathrm{~m}$, which shows a large amount of detail and structure within the lower stratosphere. The resolution drops towards lower altitudes, but stays well below $1 \mathrm{~km}$ for CFC-11, ozone and $\mathrm{HNO}_{3}$. Being able to provide such a high vertical resolution enables the reproduction of very thin filaments and will become even more valuable in combination with true 3-D reproductions of atmospheric structures.

Comparing our results with measurements of in situ instruments, we showed good agreement between the ascent profile taken by HAGAR and FOZAN during the ascent of the M55-Geophysica and a horizontally interpolated CRISTA$\mathrm{NF}$ profile measuring roughly the same air mass for ozone and CFC-11. Also the absolute values taken at flight level by the in situ instruments agree within expectations with the corresponding retrieved trace gas volume mixing ratios as derived for the same altitude from the respective CRISTANF profiles. Discrepancies largely appear close to strong increases and decreases in trace gas volume mixing ratio; this suggests that the assumption of horizontal homogeneity in trace gas volume mixing ratio along the LOS is not valid for these locations and some kind of averaging in this direction is performed by the instrument and the retrieval process. Overall, the results for these gases seem to be quantitatively reliable within the supplied error bars.

A second validation was performed with respect to profiles derived by MIPAS-STR. Here, we compared the results for five trace gases, which were derived by both instruments: ozone, CFC-11, $\mathrm{HNO}_{3}, \mathrm{ClONO}_{2}$ and $\mathrm{H}_{2} \mathrm{O}$. Only a single typical profile was discussed. Both instruments largely detected the same vertical structure in the examined trace gases, whereby MIPAS-STR, by design, is less sensitive to fine structures and tends to generate smoother profiles. The derived profiles for $\mathrm{HNO}_{3}$ also agree with one another within error bars, but they show a slightly larger trace gas volume mixing ratio for the CRISTA-NF instrument.

This suggests that the fine structures detected by the CRISTA-NF instrument are qualitatively reliable and also quantitatively sound at least for ozone and $\mathrm{CFC}-11 . \mathrm{HNO}_{3}$ might be slightly biased towards larger volume mixing ratios, but not more than suggested by the supplied diagnostics.

The CRISTA-NF instrument performed well during the campaign. The evaluation of one flight was presented and very fine vertical structures and filaments were resolved due to an unprecedented vertical resolution. Our study demonstrates the potential of the limb-sounding technique to obtain a vertical resolution of $500 \mathrm{~m}$ or better for a variety of atmospheric species if the instrument provides an adequate combination of vertical sampling and small field-of-view. This is important input for performance studies of future limbimaging satellite missions. As the well-resolved trace gases CFC-11, ozone and $\mathrm{HNO}_{3}$ are tracers for polar and midlatitude air, the CRISTA-NF dataset offers a good opportunity to study exchange processes at the edge of the polar vortex and denitrification processes in polar air.

Acknowledgements. The RECONCILE project is funded under the European Commission Seventh Framework Programme (FP7) under grant number RECONCILE-226365-FP7-ENV-2008-1.

We thank the RECONCILE coordination and flight planning teams, MDB and Enviscope for the successful implementation of and support during the Arctic field campaign.

Edited by: A. Lambert

\section{References}

Bernath, P. F., McElroy, C. T., Abrams, M. C., Boone, C. D., Butler, M., Camy-Peyret, C., Carleer, M., Clerbaux, C., Coheur, P.F., Colin, R., DeCola, P., DeMazière, M., Drummond, J. R., Dufour, D., Evans, W. F. J., Fast, H., Fussen, D., Gilbert, K., Jennings, D. E., Llewellyn, E. J., Lowe, R. P., Mahieu, E., McConnell, J. C., McHugh, M., McLeod, S. D., Michaud, R., Midwinter, C., Nassar, R., Nichitiu, F., Nowlan, C., Rinsland, C. P., Rochon, Y. J., Rowlands, N., Semeniuk, K., Simon, P., Skelton, R., Sloan, J. J., Soucy, M.-A., Strong, K., Tremblay, P., Turnbull, D., Walker, K. A., Walkty, I., Wardle, D. A., Wehrle, V., Zander, R., and Zou, J.: Atmospheric Chemistry Experiment (ACE): Mission overview, Geophys. Res. Lett., 32, L15S01, doi:10.1029/2005GL022386, 2005.

Curtis, A. R.: Discussion of "A statistical model for water vapour absorption” by Goody, R. M., Q. J. Roy. Meteor. Soc., 78, 638640, 1952.

Dee, D. P., Uppala, S. M., Simmons, A. J., Berrisford, P., Poli, P., Kobayashi, S., Andrae, U., Balmaseda, M. A., Balsamo, G., Bauer, P., Bechtold, P., Beljaars, A. C. M., van de Berg, L., Bidlot, J., Bormann, N., Delsol, C., Dragani, R., Fuentes, M., Geer, A. J., Haimberger, L., Healy, S. B., Hersbach, H., Hólm, E. V., Isaksen, L., Kållberg, P., Köhler, M., Matricardi, M., McNally, A. P., Monge-Sanz, B. M., Morcrette, J.-J., Park, B.K., Peubey, C., de Rosnay, P., Tavolato, C., Thépaut, J.-N., and Vitart, F.: The ERA-Interim reanalysis: configuration and performance of the data assimilation system, Q. J. Roy. Meteor. Soc., 137, 553-597, doi:10.1002/qj.828, 2011. 
Drummond, J. R., Houghton, J. T., Peskett, G. D., Rodgers, C. D., Wale, M. J., Whitney, J., and Williamson, E. J.: The stratospheric and mesospheric sounder on Nimbus 7, Philos. T. R. Soc. A, 296, 219-241, doi:10.1098/rsta.1980.0166, 1980.

Dudhia, A., Morris, P. E., and Wells, R. J.: Fast monochromatic radiative transfer calculations for limb sounding, J. Quant. Spectrosc. Ra., 74, 745-756, doi:10.1016/S0022-4073(01)00285-0, 2002.

Dufour, G., Boone, C. D., and Bernath, P. F.: First measurements of CFC-113 and HCFC-142b from space using ACE-FTS infrared spectra, Geophys. Res. Lett., 32, L15S09, doi:10.1029/2005GL022422, 2005.

Eckermann, S. D., Hoffmann, L., Höpfner, M., Wu, D. L., and Alexander, M. J.: Antarctic NAT PSC belt of June 2003: observational validation of the mountain wave seeding hypothesis, Geophys. Res. Lett., 36, L02807, doi:10.1029/2008GL036629, 2009.

ESA: Candidate Earth Explorer Core Missions - Report for Assessment: PREMIER - PRocess Exploitation through Measurements of Infrared and millimetre-wave Emitted Radiation, vol. SP-1313/5, ESA Publications Division, ESTEC, Keplerlaan 1, 2200 AG Noordwijk, The Netherlands, 2008.

Fastie, W.: Ebert spectrometer reflections, Phys. Today, 4, 37-43, 1991.

Fischer, H., Birk, M., Blom, C., Carli, B., Carlotti, M., von Clarmann, T., Delbouille, L., Dudhia, A., Ehhalt, D., Endemann, M., Flaud, J. M., Gessner, R., Kleinert, A., Koopman, R., Langen, J., López-Puertas, M., Mosner, P., Nett, H., Oelhaf, H., Perron, G., Remedios, J., Ridolfi, M., Stiller, G., and Zander, R.: MIPAS: an instrument for atmospheric and climate research, Atmos. Chem. Phys., 8, 2151-2188, doi:10.5194/acp-8-2151-2008, 2008.

Flaud, J.-M., Brizzi, G., Carlotti, M., Perrin, A., and Ridolfi, M.: MIPAS database: Validation of $\mathrm{HNO}_{3}$ line parameters using MIPAS satellite measurements, Atmos. Chem. Phys., 6, 5037-5048, doi:10.5194/acp-6-5037-2006, 2006.

Francis, G. L., Edwards, D. P., Lambert, A., Halvorson, C. M., Lee-Taylor, J. M., and Gille, J. C.: Forward modeling and radiative transfer for the NASA EOS-Aura High Resolution Dynamics Limb Sounder (HIRDLS) instrument, J. Geophys. Res., 111, D13301, doi:10.1029/2005JD006270, 2006.

Friedl-Vallon, F., Riese, M., Maucher, G., Lengel, A., Hase, F., Preusse, P., and Spang, R.: Instrument concept and preliminary performance analysis of GLORIA, Adv. Space Res., 37, 22872291, doi:10.1016/j.asr.2005.07.075, 2006.

Gettelman, A., Hoor, P., Pan, L. L., Randell, W. J., Hegglin, M. I., and Birner, T.: The extra tropical upper troposphere and lower stratosphere, Rev. Geophys., 49, RG3003, doi:10.1029/2011RG000355, 2011.

Gille, J. C. and Russel III, J. M.: The limb infrared monitor of the stratosphere: experiment description, performance, and results, J. Geophys. Res., 89, 5125-5140, doi:10.1029/JD089iD04p05125, 1984.

Glatthor, N., von Clarmann, T., Fischer, H., Funke, B., Grabowski, U., Höpfner, M., Kellmann, S., Kiefer, M., Linden, A., Milz, M., Steck, T., and Stiller, G. P.: Global peroxyacetyl nitrate (PAN) retrieval in the upper troposphere from limb emission spectra of the Michelson Interferometer for Passive Atmospheric Sounding (MIPAS), Atmos. Chem. Phys., 7, 27752787, doi:10.5194/acp-7-2775-2007, 2007.
Godson, W. L.: The evaluation of infra-red radiative fluxes due to atmospheric water vapour, Q. J. Roy. Meteor. Soc., 79, 367-379, 1953.

Gordley, L. L. and Russell, J. M.: Rapid inversion of limb radiance data using an emissivity growth approximation, Appl. Opt., 20, 807-813, doi:10.1364/AO.20.000807, 1981.

Grooß, J.-U., Günther, G., Müller, R., Konopka, P., Bausch, S., Schlager, H., Voigt, C., Volk, C. M., and Toon, G. C.: Simulation of denitrification and ozone loss for the Arctic winter 2002/2003, Atmos. Chem. Phys., 5, 1437-1448, doi:10.5194/acp-5-14372005, 2005.

Grossmann, K. U., Offermann, D., Gusev, O., Oberheide, J., Riese, M., and Spang, R.: The CRISTA-2 mission, J. Geophys. Res., 107, D23, doi:10.1029/2001JD000667, 2002.

Hase, F. and Höpfner, M.: Atmospheric ray path modeling for radiative transfer algorithms, Appl. Opt., 38, 3129-3133, doi:10.1364/AO.38.003129, 1999.

Hoffmann, L. and Alexander, M. J.: Retrieval of stratospheric temperatures from atmospheric infrared sounder radiance measurements for gravity wave studies, J. Geophys. Res., 114, D07105, doi:10.1029/2008JD011241, 2009.

Hoffmann, L., Kaufmann, M., Spang, R., Müller, R., Remedios, J. J., Moore, D. P., Volk, C. M., von Clarmann, T., and Riese, M.: Envisat MIPAS measurements of CFC-11: retrieval, validation, and climatology, Atmos. Chem. Phys., 8, 3671-3688, doi:10.5194/acp-8-3671-2008, 2008.

Hoffmann, L., Weigel, K., Spang, R., Schroeder, S., Arndt, K., Lehmann, C., Kaufmann, M., Ern, M., Preusse, P., Stroh, F., and Riese, M.: CRISTA-NF measurements of water vapor during the SCOUT-O $_{3}$ Tropical Aircraft Campaign, Adv. Space Res., 43, 74-81, doi:10.1016/j.asr.2008.03.018, 2009.

Höpfner, M., Blom, C., Clarmann, T., Fischer, F., Glatthor, N., Gulde, T., Hase, F., Keim, C., Kimmig, W., Lessenich, K., Piesch, C., Sartorius, C., and Stiller, G.: MIPAS-STR data analysis of APE-GAIA measurements, in: IRS 2000: Current Problems in Atmospheric Radiation; Proc. of the Internat. Radiation Symp., edited by: Smith, W., 1136-1139, DEEPAK Publ., St. Petersburg, Russia, 2000.

Kalicinsky, C., Grooss, J.-U., Guenther, G., Ungermann, J., Olschewski, F., Knieling, P., Hoefer, S., Blank, J., Stroh, F., and Riese, M.: CRISTA-NF observations of atmospheric mixing processes in the vicinity of the polar vortex, in preparation.

Keim, C.: Entwicklung und Verifikation der Sichtlinienstabilisierung für MIPAS auf dem hochfliegenden Forschungsflugzeug M55 Geophysica, Wissenschaftliche Berichte, FZKA, 6729, Dissertation, Universität Karlsruhe, Karlsruhe, 2002.

Keim, C., Liu, G. Y., Blom, C. E., Fischer, H., Gulde, T., Höpfner, M., Piesch, C., Ravegnani, F., Roiger, A., Schlager, H., and Sitnikov, N.: Vertical profile of peroxyacetyl nitrate (PAN) from MIPAS-STR measurements over Brazil in February 2005 and its contribution to tropical UT $\mathrm{NO}_{\mathrm{y}}$ partitioning, Atmos. Chem. Phys., 8, 4891-4902, doi:10.5194/acp-8-4891-2008, 2008.

Kullmann, A., Riese, M., Olschewski, F., Stroh, F., and Grossmann, K. U.: Cryogenic infrared spectrometers and telescopes for the atmosphere - new frontiers, Proc. SPIE, 5570, 423-432, 2004.

Offermann, D., Grossmann, K.-U., Barthol, P., Knieling, P., Riese, M., and Trant, R.: Cryogenic Infrared Spectrometers and 
Telescopes for the Atmosphere (CRISTA) experiment and middle atmosphere variability, J. Geophys. Res., 104, 16311-16325, doi:10.1029/1998JD100047, 1999.

Piesch, C., Gulde, T., Sartorius, C., Friedl-Vallon, F., Seefeldner, M., Wölfel, M., Blom, C., and Fischer, H.: Design of a MIPAS instrument for high-altitude aircraft, in: Proc. of the Second Internat. Airborne Remote Sensing Conference and Exhibition, Vol. II, 199-208, Ann Arbor, MI, USA, 1996.

Remedios, J. J., Leigh, R. J., Waterfall, A. M., Moore, D. P., Sembhi, H., Parkes, I., Greenhough, J., Chipperfield, M. P., and Hauglustaine, D.: MIPAS reference atmospheres and comparisons to V4.61/V4.62 MIPAS level 2 geophysical data sets, Atmos. Chem. Phys. Discuss., 7, 9973-10017, doi:10.5194/acpd-79973-2007, 2007.

Riediger, O., Volk, C. M., Strunk, M., and Schmidt, U.: HAGAR - a new in situ tracer instrument for stratospheric balloons and high altitude aircraft, Eur. Comm. Air Pollut. Res. Report, 73, 727-730, 2000.

Riese, M., Preusse, P., Spang, R., Ern, M., Jarisch, M., Grossmann, U., and Offermann, D.: Measurements of trace gases by the cryogenic infrared spectrometers and telescopes for the atmosphere CRISTA experiment, Adv. Space Res., 19, 563-566, doi:10.1016/S0273-1177(97)00172-5, 1997.

Riese, M., Spang, R., Preusse, P., Ern, M., Jarisch, M., Offermann, D., and Grossmann, K. U.: Cryogenic Infrared Spectrometers and Telescopes for the Atmosphere (CRISTA) data processing and atmospheric temperature and trace gas retrieval, J. Geophys. Res., 104, 16349-16367, doi:10.1016/S0273-1177(97)00172-5, 1999a.

Riese, M., Tie, X., Brasseur, G., and Offermann, D.: Threedimensional simulation of stratospheric trace gas distributions measured by CRISTA, J. Geophys. Res., 104, 16419-16435, doi:10.1029/1999JD900178, 1999b.

Riese, M., Manney, G. L., Oberheide, J., Tie, X., Spang, R., and Küll, V.: Stratospheric transport by planetary wave mixing as observed during CRISTA-2, J. Geophys. Res., 107, 8179, doi:10.1029/2001JD000629, 2002.

Riese, M., Friedl-Vallon, F., Spang, R., Preusse, P., Schiller, C., Hoffmann, L., Konopka, P., Oelhaf, H., von Clarmann, T., and Höpfner, M.: GLObal limb Radiance Imager for the Atmosphere (GLORIA): scientific objectives, Adv. Space Res., 36, 989-995, doi:10.1016/j.asr.2005.04.115, 2005.

Rodgers, C. D.: Inverse Methods for Atmospheric Sounding: Theory and Practice, World Scientific, 2000.

Rothman, L., Gordon, I., Barbe, A., Benner, D., Bernath, P., Birk, M., Boudon, V., Brown, L., Campargue, A., Champion, J.-P., Chance, K., Coudert, L., Dana, V., Devi, V., Fally, S., Flaud, J.-M., Gamache, R., Goldman, A., Jacquemart, D., Kleiner, I., Lacome, N., Lafferty, W., Mandin, J.-Y., Massie, S., Mikhailenko, S., Miller, C., Moazzen-Ahmadi, N., Naumenko, O., Nikitin, A., Orphal, J., Perevalov, V., Perrin, A., Predoi-Cross, A., Rinsland, C., Rotger, M., Simecková, M., Smith, M., Sung, K., Tashkun, S., Tennyson, J., Toth, R., Vandaele, A., and Auwera, J. V.: The HITRAN 2008 molecular spectroscopic database, J. Quant. Spectrosc. Ra., 110, 533-572, doi:10.1016/j.jqsrt.2009.02.013, 2009.

Schroeder, S., Kullman, A., Preusse, P., Stroh, F., Weigel, K., Ern, M., Knieling, P., Olschewski, F., Spang, R., and Riese, M.: Radiance calibration of CRISTA-NF, Adv. Space Res., 43, 1910-
1917, doi:10.1016/j.asr.2009.03.009, 2009.

Spang, R., Hoffmann, L., Kullmann, A., Olschewski, F., Preusse, P., Knieling, P., Schroeder, S., Stroh, F., Weigel, K., and Riese, M.: High resolution limb observations of clouds by the CRISTA-NF experiment during the SCOUT-O3 tropical aircraft campaign, Adv. Space Res., 42, 1765-1775, doi:10.1016/j.asr.2007.09.036, 2008.

Steck, T. and von Clarmann, T.: Constrained profile retrieval applied to the observation mode of the Michelson Interferometer for Passive Atmospheric Sounding, Appl. Opt., 40, 3559-3571, doi:10.1364/AO.40.003559, 2001.

Tikhonov, A. N. and Arsenin, V. Y.: Solutions of ill-posed problems, Winston, Washington, DC, USA, 1977.

Twomey, S.: Introduction to the Mathematics of Inversion in Remote Sensing and Indirect Measurements, Dover Publications, Mineola, NY, USA, 1977.

Ulanovsky, A. E., Yushkov, V. A., Sitnikov, N. M., and Ravengnani, F.: The FOZAN-II fast-response chemiluminescent airborne ozone analyzer, Instrum. Exp. Tech., 44, 249-256, doi:10.1023/A:1017535608026, 2001.

Ungermann, J.: Tomographic reconstruction of atmospheric volumes from infrared limb-imager measurements, Ph.D. Thesis, Wuppertal University, Forschungszentrum Jülich, Jülich, 2011.

Ungermann, J., Kaufmann, M., Hoffmann, L., Preusse, P., Oelhaf, H., Friedl-Vallon, F., and Riese, M.: Towards a 3-D tomographic retrieval for the air-borne limb-imager GLORIA, Atmos. Meas. Tech., 3, 1647-1665, doi:10.5194/amt-3-1647-2010, 2010.

Ungermann, J., Blank, J., Lotz, J., Leppkes, K., Guggenmoser, T., Kaufmann, M., Preusse, P., Naumann, U., and Riese, M.: A 3-D tomographic trajectory retrieval for the air-borne limbimager GLORIA, Atmos. Meas. Tech. Discuss., 4, 3805-3859, doi:10.5194/amtd-4-3805-2011, 2011.

von Clarmann, T., De Clercq, C., Ridolfi, M., Hölpfner, M., and Lambert, J.-C.: The horizontal resolution of MIPAS, Atmos. Meas. Tech., 2, 47-54, doi:10.5194/amt-2-47-2009, 2009.

Weigel, K., Riese, M., Hoffmann, L., Hoefer, S., Kalicinsky, C., Knieling, P., Olschewski, F., Preusse, P., Spang, R., Stroh, F., and Volk, C. M.: CRISTA-NF measurements during the AMMASCOUT-O3 aircraft campaign, Atmos. Meas. Tech., 3, 14371455, doi:10.5194/amt-3-1437-2010, 2010.

Weinreb, M. P. and Neuendorffer, A. C.: Method to apply homogeneous-path transmittance models to inhomogeneous atmospheres, J. Atmos. Sci., 30, 662-666, doi:10.1175/15200469(1973)030<0662:MTAHPT>2.0.CO;2, 1973.

Werner, A., Volk, C. M., Ivanova, E. V., Wetter, T., Schiller, C., Schlager, H., and Konopka, P.: Quantifying transport into the Arctic lowermost stratosphere, Atmos. Chem. Phys., 10, 1162311639, doi:10.5194/acp-10-11623-2010, 2010.

Woiwode, W., Oelhaf, H., Gulde, T., Piesch, C., Maucher, G., Ebersoldt, A., Keim, C., Höpfner, M., Khaykin, S., Ravegnani, F., Ulanovsky, A. E., Volk, C. M., Hösen, E., Dörnbrack, A., Ungermann, J., Kalicinsky, C., and Orphal, J.: MIPAS-STR measurements in the arctic UTLS in winter/spring 2010: instrument characterization, retrieval and validation, Atmos. Meas. Tech. Discuss., 4, 7035-7108, doi:10.5194/amtd-4-7035-2011, 2011. 